\title{
Enhanced transverse shear strain shell formulation applied to large elasto-plastic deformation problems
}

\author{
R. A. Fontes Valente ${ }^{1,2, *, \dagger}$, M. P. L. Parente ${ }^{2}$, R. M. Natal Jorge ${ }^{2}$, \\ J. M. A. César de á $^{2}$ and J. J. Grácio ${ }^{1}$ \\ ${ }^{1}$ Department of Mechanical Engineering, University of Aveiro, Aveiro, Portugal \\ ${ }^{2}$ IDMEC, Faculty of Engineering, University of Porto, Porto, Portugal
}

\begin{abstract}
SUMMARY
In this work, a previously proposed Enhanced Assumed Strain (EAS) finite element formulation for thin shells is revised and extended to account for isotropic and anisotropic material non-linearities. Transverse shear and membrane-locking patterns are successfully removed from the displacement-based formulation. The resultant EAS shell finite element does not rely on any other mixed formulation, since the enhanced strain field is designed to fulfil the null transverse shear strain subspace coming from the classical degenerated formulation. At the same time, a minimum number of enhanced variables is achieved, when compared with previous works in the field. Non-linear effects are treated within a local reference frame affected by the rigid-body part of the total deformation. Additive and multiplicative update procedures for the finite rotation degrees-of-freedom are implemented to correctly reproduce mid-point configurations along the incremental deformation path, improving the overall convergence rate. The stress and strain tensors update in the local frame, together with an additive treatment of the EAS terms, lead to a straightforward implementation of non-linear geometric and material relations. Accuracy of the implemented algorithms is shown in isotropic and anisotropic elasto-plastic problems. Copyright (C) 2004 John Wiley \& Sons, Ltd.
\end{abstract}

KEY WORDS: shell element; enhanced transverse shear strain; transverse shear locking; large rotations; large deformations; anisotropic elasto-plasticity

\footnotetext{
${ }^{*}$ Correspondence to: R. A. Fontes Valente, Department of Mechanical Engineering, University of Aveiro, Campus de Santiago, 3810-193 Aveiro, Portugal.

†E-mail: robertt@mec.ua.pt
}

Contract/grant sponsor: Ministério da Ciência e do Ensino Superior (FCT and FSE), Portugal; contract/grant number: PARXIS XXI/BD/21662/99

Contract/grant sponsor: FEDER Program, Portugal; contract/grant numbers: POCTI/EME/47289/2002 and POSI SFRH/BD/13013/2003

Copyright (c) 2004 John Wiley \& Sons, Ltd.

Received 23 December 2003

Revised 15 September 2004

Accepted 15 September 2004 


\section{INTRODUCTION}

Finite shell elements technology started with the development of the so-called 'degeneration approach', early introduced by Ahmad et al. [1], where a class of shell elements were obtained from the continuum, resorting to the concept of a 'reference' surface. Plane-stress constitutive conditions have automatically been included into the formulation, in a way to make elements numerically consistent with the modelling of thin structures. Since then, isoparametric shell elements incorporating displacement and rotation-like degrees-of-freedom have been continuously improved, with extensions to different kinds of problems being presented, for instance, by Krakeland [2], Ramm [3], Zienkiewicz et al. [4], Bathe and Bolourchi [5], Hughes and Liu [6], Hughes and Tezduyar [7] and Liu et al. [8], just to name the earliest works. A 'complete' list of relevant contributions would be extensive (if feasible) and beyond the scope of this paper. Nevertheless, a comprehensive study on the evolution of shell elements technology can be reported in the prominent work of Yang et al. [9].

Sooner, however, it was verified that this class of elements, particularly the bilinear ones (treated in the present work), was prone to show locking effects in the thin shell limit. For this case, transverse shear strain energy did not vanish at all points within the element domain for pure bending deformations, contradicting the Kirchhoff-Koiter-Love hypothesis for membrane structures. The situation is even worse as it is clearly difficult to establish a definite boundary between situations where a given formulation tends to 'lock' or not, with locking appearance being not just a matter of thickness values (or ratio between thickness and a dominant dimension), as recently demonstrated by Chapelle, Bathe and co-workers [10-15].

In order to circumvent transverse shear locking phenomena, selective reduced integration technique [16,4], mixed interpolation of tensorial components [17,18] (with roots in earlier publications $[19,7,20]$ ) and, finally, the assumed natural strain [21-23] have proved to he successful approaches. The common point with these formulations is the fact that the original, displacement-based, strain field is judiciously replaced by substituting components which are interpolated in distinct (non-conventional) ways.

The Enhanced Assumed Strain (EAS) method, from the classical set of papers by Simo, Rifai, Armero and Taylor [24-26], departs from the previous works, keeping the original strain field unchanged, then adding additional strain terms, chosen in conformity to the desired improvement in a given element's formulation. In this way, the same formalism can be applied to a set of pathologies appearing in lower-order finite element formulations, such as the in-plane locking (2D elements), the volumetric locking (3D elements) or membrane locking. Focusing on the membrane locking, but applied to plate and shell elements, first works dealing with the enhanced assumed strain concept were presented by Andelfinger and Ramm [27] and Büchter et al. [28]. About the specific case of transverse shear locking in thin shells, Simo and Rifai [24] have also introduced a proposal with, however, no strong usage in the literature afterwards.

This was the starting point for the work of the authors. Departing from the conventional degenerated approach applied to bilinear (four-node) fully integrated shell elements, a complete analysis of the null transverse shear strain subspace was performed in Reference [29]. Along with the present work, this reference points to previous developments by the authors in EASbased two dimension, shell and solid-shell finite elements technology [30-36].

For the specific case of shell elements, the likelihood of transverse shear locking to happen was related to the ability (or not) of a given formulation to successfully reproduce the correct solution by naturally providing enough components to the subspace basis [29]. The main 
advantage of this subspace analysis was that its validity remains unaltered irrespective of the loading and boundary conditions applied to a given element, thus being a characteristic of a given formulation. Comparison of the subspace bases provided by the degenerated approach, the selective reduced integration method and the mixed interpolation of tensorial components have revealed missing components in the first formulation. Instead of performing a replacement of transverse shear strain terms to correct the element formulation, the EAS method was then used to additively improve the transverse shear strain field coming from the displacement-based formulation. This has led to a set of shell elements with distinct number of internal variables and with no transverse shear locking for a variety of linear test cases [29]. A preliminary study on large rotation problems, while keeping elastic strains, was performed by the authors in Reference [32] with encouraging results.

In the present work, the previous shell formulation is revised and improved, from both the fundamental and computational aspects. Comparatively, the approach here presented allows the use of a lower number of EAS internal variables, with gains of computational time and efficiency. An exhaustive set of results coming from linear and non-linear benchmarks is detailed in the report [33], with its main aspects and conclusions being summarized in the present paper. In doing so, this study accomplishes for results coming from both smooth and non-smooth shell structures, while accounting for non-linear geometric and material models. Related to the latter topic, isotropic as well as planar and normal anisotropic yield criteria are implemented. Results obtained with the shell formulation, along with the adopted constitutive laws, are compared with those provided by experimental and simulation data from well-established formulations in the literature.

\section{SHELL DISPLACEMENT-BASED KINEMATICS}

In dealing with shell formulations directly derived from the degenerated concept [1], it is commonplace to assume the existence of a reference surface $\mathscr{B} \subset \mathbb{R}^{3}$, with boundary $\partial \mathscr{B}$, which can be occupied by a given shell element. Considering the deformation of the continuum, it is also useful to invoke a converged (material) and current (spatial) configurations $\mathscr{M} \subset \mathbb{R}^{3}$ and $\mathscr{S} \subset \mathbb{R}^{3}$, respectively. In static analysis, these two configurations mimic successive deformation stages $(n)$ and $(n+1)$ of a given body partition, without resorting to the time variable.

Departing from a given continuum body into a discretized one, finite elements can be described within a set of curvilinear (convective) co-ordinates

$$
\xi=\left(\xi^{1}, \xi^{2}, \xi^{3}\right) \equiv[-1,1] \times[-1,1] \times[-1,1]
$$

characterizing a parametric configuration $\mathscr{P} \subset \mathbb{R}^{3}$. Related to this parametric set, the position vector of any point $P \in \mathscr{B}$ of a shell element can then be defined by $[37,15]$

$$
{ }^{n} \mathbf{x}(\boldsymbol{\xi})=\mathbf{N}_{k}{ }^{n} \mathbf{x}_{k}+\frac{1}{2} \xi^{3 n} a_{k} \mathbf{N}_{k}{ }^{n} \mathbf{v}_{k}^{3}
$$

for configuration $(n)$. In this equation, summation over indices $k$ (nodes) is implicit. Nodes are assumed to rely on the (reference) mid-surface, being ${ }^{n} \mathbf{x}_{k}$ (nodal) position vectors related to an external system of orthonormal vectors, forming a global triad $\left(\mathbf{e}_{1}, \mathbf{e}_{2}, \mathbf{e}_{3}\right)$. Also in Equation (2), $\mathbf{N}_{k}=\mathbf{N}_{k}\left(\xi^{1}, \xi^{2}\right)$ are the matrices of two-dimensional isoparametric shape functions, ${ }^{n} a_{k}$ are the nodal thickness values and ${ }^{n} \mathbf{v}_{k}^{3}$ represents a (unit) director vector, normal (at least in 
the initial state) to a plane formed by the tangent to the convective axes $\left(\xi^{1}, \xi^{2}\right)$, and defining the so-called thickness direction.

In detail, for a mid-surface in the $\left(\xi^{1}, \xi^{2}\right)$ space, and mapped onto $\mathscr{B}$ via a function (chart) $\phi\left(\xi^{1}, \xi^{2}\right)$, a couple of auxiliary (tangent) vectors can be defined in a general form as [15]

$$
\mathbf{v}_{l}^{\alpha}=\frac{\partial \phi\left(\xi^{1}, \xi^{2}\right)}{\partial \xi^{\alpha}} \quad \text { for } \alpha=1,2
$$

By assumption, these linearly independent vectors are candidates in forming a frame on the plane tangent to the mid-surface at each point $(l)$. The director vector can then be explicitly defined as

$$
\mathbf{v}_{l}^{3}=\frac{\mathbf{v}_{l}^{1} \times \mathbf{v}_{l}^{2}}{\left\|\mathbf{v}_{l}^{1} \times \mathbf{v}_{l}^{2}\right\|}
$$

However, in algorithmic terms and for a nodal point $(l=k)$ within a finite element, the nodal director can be defined, for instance, from the global co-ordinates of adjacent nodes or directly from the user input [38], in order to coincide (or be close) its direction to the fibre (thickness) direction [39]. Unit vectors $\left(\mathbf{v}_{k}^{1}, \mathbf{v}_{k}^{2}, \mathbf{v}_{k}^{3}\right)$ on each node then introduce a unique nodal co-ordinate system at each configuration.

After deformation, the position of point $P$ can be described in the final configuration $(n+1)$ as

$$
{ }^{n+1} \mathbf{x}(\xi)=\mathbf{N}_{k}{ }^{n+1} \mathbf{x}_{k}+\frac{1}{2} \xi^{3 n+1} a_{k} \mathbf{N}_{k}^{n+1} \mathbf{v}_{k}^{3}
$$

The displacement field of a given point $\left(\xi^{1}, \xi^{2}, \xi^{3}\right)$ between configurations $(n)$ and $(n+1)$ now appears as

$$
{ }_{n}^{n+1} \mathbf{u}(\xi)={ }^{n+1} \mathbf{x}(\xi)-{ }^{n} \mathbf{x}(\xi)
$$

Expansion of Equation (6) gives rise to five nodal degrees-of-freedom, comprising three translations related to the global frame $\left(\mathbf{e}_{1}, \mathbf{e}_{2}, \mathbf{e}_{3}\right)$ plus two rotations related to the nodal frame $\left(\mathbf{v}_{k}^{1}, \mathbf{v}_{k}^{2}, \mathbf{v}_{k}^{3}\right)$ and coming from the general expression

$$
{ }_{n}^{n+1} \mathbf{u}(\boldsymbol{\xi})=\mathbf{N}_{k}{ }_{n}^{n+1} \mathbf{x}+\frac{1}{2} \xi^{3} \mathbf{N}_{k}\left({ }^{n+1} a_{k}{ }^{n+1} \mathbf{v}_{k}^{3}-{ }^{n} a_{k}{ }^{n} \mathbf{v}_{k}^{3}\right)
$$

In the present work, from configurations $(n)$ to $(n+1)$, nodal thickness values are kept constant and equal to $a_{k}={ }^{n} a_{k}={ }^{n+1} a_{k}$. After the completion of iterations until the final stage $(n+1)$, thickness values at Gauss points are updated enforcing the plane-stress condition, usual in this class of degenerated shell elements. These Gaussian thickness values are subsequently extrapolated to the nodal points, and a new deformation stage is started with the corrected nodal thickness values $[40,41]$.

Apart from these considerations about nodal thickness values, nodal director variations in Equation (7) can be explicitly described in the nodal frame $\left(\mathbf{v}_{k}^{1}, \mathbf{v}_{k}^{2}, \mathbf{v}_{k}^{3}\right)$ as

$$
{ }_{n}^{n+1} \mathbf{v}_{k}^{3}={ }^{n+1} \mathbf{v}_{k}^{3}-{ }^{n} \mathbf{v}_{k}^{3}=\theta_{k}^{1{ }^{n}} \mathbf{v}_{k}^{1}-\theta_{k}^{2 n} \mathbf{v}_{k}^{2}-\frac{1}{2}\left[\left(\theta_{k}^{1}\right)^{2}+\left(\theta_{k}^{2}\right)^{2}\right]{ }^{n} \mathbf{v}_{k}^{3}
$$

where $\left(\theta_{k}^{1}, \theta_{k}^{2}\right)$ are the rotational degrees-of-freedom of director ${ }^{n} \mathbf{v}_{k}^{3}$ about auxiliary vectors ${ }^{n} \mathbf{v}_{k}^{1}$ and ${ }^{n} \mathbf{v}_{k}^{2}$, respectively. Although valid for large incremental rotations, the contribution of the 
quadratic terms in Equation (8) to the displacement-based geometric stiffness matrix is not accounted for in the present work, without prejudice of the overall convergence behaviour and with the benefit of the computational costs, in the same manner as stated in Reference [42].

The rotational degrees-of-freedom $\left(\theta_{k}^{1}, \theta_{k}^{2}\right)$ are also used in the construction of a rotationbased matrix, responsible for the update of the nodal triad at each iteration between two successive load levels $(n)$ and $(n+1)$. Focusing on the current and converged directors at a node, it is possible to state that

$$
\left.{ }^{n+1} \mathbf{v}_{k}^{3}\right|^{i}={ }_{n}^{n+1} \boldsymbol{\Lambda}_{k}^{i}{ }^{n} \mathbf{v}_{k}^{3}
$$

for a given iteration $(i)$. The general form of the second-order transformation tensor adopted in this work is the Rodrigues formula from rigid body dynamics [43-46]

$$
{ }_{n}^{n+1} \boldsymbol{\Lambda}_{k}^{i} \equiv \mathrm{e}^{\boldsymbol{\Phi}_{k}^{i}}=\mathbf{I}_{2}+\frac{\sin (\phi)}{\phi} \boldsymbol{\Phi}_{k}^{i}+\frac{1-\cos (\phi)}{\phi^{2}} \boldsymbol{\Phi}_{k}^{i} \boldsymbol{\Phi}_{k}^{i}
$$

for the second-order identity tensor $\mathbf{I}_{2}$. In this equation, $\boldsymbol{\Phi}_{k}^{i}$ contains a composition of the nodal rotational degrees of freedom $\theta_{k}^{1}$ and $\theta_{k}^{2}$, from $(n)$ to the $i$ th iteration up to $(n+1)$, in the form

$$
\left[\boldsymbol{\Phi}_{k}^{i}\right]=\left[\begin{array}{ccc}
0 & 0 & { }_{n}^{i} \theta_{k}^{1} \\
0 & 0 & -{ }_{n}^{i} \theta_{k}^{2} \\
-{ }_{n}^{i} \theta_{k}^{1} & { }_{n}^{i} \theta_{k}^{2} & 0
\end{array}\right] \text { and with } \phi=\sqrt{\left({ }_{n}^{i} \theta_{k}^{1}\right)^{2}+\left({ }_{n}^{i} \theta_{k}^{2}\right)^{2}}
$$

once no drilling degrees-of-freedom are employed in the present formulation.

The converged director $\left({ }^{n} \mathbf{v}_{k}^{3}\right)$ is taken as the reference one, being updated at each iteration (i), and leading to a 'pseudo-converged' current director $\left({ }^{n+1} \mathbf{v}_{k}^{3} i^{i}\right)$ with iterative character. This involves the knowledge of the total values of the rotational degrees-of-freedom $\left({ }_{n}^{i} \theta_{k}^{1},{ }_{n}^{i} \theta_{k}^{2}\right)$ from the converged state $(n)$ up to the current iteration $(i)$, respectively. Since the degrees-of-freedom $\left(\theta_{k}^{1}, \theta_{k}^{2}\right)$ are, by definition, non-additive variables (even for a small deformation analysis), a special procedure to convert them into additive variables is employed, following the general guidelines of Crisfield [47] and detailed in the following for the sake of completeness.

Consider the accumulated rotation vector (previously additively evaluated) from converged state $(n)$ until the previous iteration $(i-1)$ for a given node as

$$
{ }_{n}^{i-1} \boldsymbol{\theta}_{k}=\sum_{j=1}^{i-1}{ }_{j-1}^{j} \boldsymbol{\theta}_{k}^{a}=\left\{\begin{array}{c}
{ }^{i-1} \theta_{k}^{1} \\
i-1 \\
n \\
0
\end{array}\right\} ; \text { summation over } j \text { iterations }
$$

where the index $(a)$ enforces the additive character of the (transformed) rotations. If the iterative variation in the rotational variables corresponds to vector $\left({ }_{i-1}^{i} \boldsymbol{\theta}_{k}\right)$, it is possible to change its non-additive character into an additive one, represented by a new iterative rotation vector $\left({ }_{i-1}^{i} \theta_{k}^{a}\right)$ in the form

$$
{ }_{i-1}^{i} \boldsymbol{\theta}_{k}^{a}=\left[\mathbf{H}\left({ }_{n}^{i-1} \boldsymbol{\theta}_{k}\right)\right]^{-1}{ }_{i-1}^{i} \boldsymbol{\theta}_{k}
$$


accounting for

$$
\mathbf{H}\left({ }_{n}^{i-1} \boldsymbol{\theta}_{k}\right)=\frac{\sin (\phi)}{\phi} \mathbf{I}_{2}+\frac{1}{\phi^{2}}\left(1-\frac{\sin (\phi)}{\phi}\right){ }_{n}^{i-1} \boldsymbol{\theta}_{k}\left[{ }_{n}^{i-1} \boldsymbol{\theta}_{k}\right]^{\mathrm{T}}+\frac{1}{2}\left(\frac{\sin (\phi / 2)}{\phi / 2}\right)^{2} \boldsymbol{\Phi}_{k}\left({ }_{n}^{i-1} \boldsymbol{\theta}_{k}\right)
$$

and where the function $\boldsymbol{\Phi}_{k}\left({ }_{n}^{i-1} \boldsymbol{\theta}_{k}\right)$ is evaluated according to Equation (11). The updated incremental additive variation can now be directly employed in the director update expressions

$$
\left({ }_{n}^{i-1} \boldsymbol{\theta}_{k}+{ }_{i-1}^{i} \boldsymbol{\theta}_{k}^{a}\right) \stackrel{\text { Equations (9),(10),(11) }}{=}\left({ }_{n}^{n+1} \boldsymbol{\Lambda}_{k}^{i}\right)
$$

The same procedure as detailed for the update of the director $\left({ }^{n} \mathbf{v}_{k}^{3}\right)$ can be extended in order to obtain the updated triad $\left({ }^{n+1} \mathbf{v}_{k}^{1},{ }^{n+1} \mathbf{v}_{k}^{2},{ }^{n+1} \mathbf{v}_{k}^{3}\right)$, at each iteration $(i)$. After these considerations about the nodal frame in each node and configuration, the displacement-based strain tensor for this class of shell elements can be completely defined.

Taking the deformation path between configurations $(n)$ and $(n+1)$ (dropping superscripts (i) for simplicity reasons), the two-point relative deformation gradient tensor

$$
{ }_{n}^{n+1} \mathbf{F}(\boldsymbol{\xi})=\frac{\partial^{n+1} \mathbf{x}}{\partial^{n} \mathbf{x}}={ }^{n+1} \mathbf{g}_{k} \otimes{ }^{n} \mathbf{g}^{k}
$$

can be defined as a function of the position vectors and involving the set of covariant and contravariant based vectors $\left({ }^{n+1} \mathbf{g}_{k}\right)$ and $\left({ }^{n} \mathbf{g}^{k}\right)$ [37, 43].

The displacement-based Green-Lagrange strain tensor $\mathbf{E}^{u}$, as well as its components, can then be stated in the form

$$
\begin{aligned}
{ }_{n}^{n+1} \mathbf{E}^{u} & =\frac{1}{2}\left({ }_{n}^{n+1} \mathbf{F}^{\mathrm{T} n+1} \mathbf{F}-\mathbf{I}_{2}\right)=E_{\xi^{j} \xi^{l}}^{u}{ }^{n} \mathbf{g}^{j} \otimes^{n} \mathbf{g}^{l} \\
{ }_{n}^{n+1} E_{\xi^{j} \xi^{l}}^{u} & =\frac{1}{2}(\underbrace{{ }^{n} \mathbf{g}_{j} \cdot \frac{\partial_{n}^{n+1} \mathbf{u}}{\partial \xi^{l}}+\frac{\partial^{n+1} \mathbf{u}}{\partial \xi^{j}} \cdot{ }^{n} \mathbf{g}_{l}}_{\text {linear part }}+\underbrace{\frac{\partial^{n+1} \mathbf{u}}{\partial \xi^{j}} \cdot \frac{\partial^{n+1} \mathbf{u}}{\partial \xi^{l}}}_{\text {non-linear part }})
\end{aligned}
$$

The choice of the convective frame as the departure point for strain components evaluations is due to generality reasons and also because the enhanced transverse shear strain field used in this work is firstly defined in this referential, as will be detailed in the next section. The first two terms of the right-hand side of Equation (17b) lead to the linear strain-displacement and, subsequently, linear stiffness matrices.

A degrees-of-freedom vector $\mathbf{d}_{k}$, gathering the translation and rotational components at each node point $(k)$, is now introduced in the form

$$
\mathbf{d}_{k}=\left\{\begin{array}{lllll}
u^{1} & u^{2} & u^{3} & \theta^{1} & \theta^{2}
\end{array}\right\}^{\mathrm{T}}
$$

for $\mathbf{u}_{k}=\left\{\begin{array}{lll}u^{1} & u^{2} & u^{3}\end{array}\right\}^{\mathrm{T}}$. Considering the director cosines of the nodal vectors $\left(\mathbf{v}_{k}^{1}, \mathbf{v}_{k}^{2}, \mathbf{v}_{k}^{3}\right)$ as $\mathbf{v}_{k}^{1}=\left\{\begin{array}{lll}v^{11} & v^{12} & v^{13}\end{array}\right\}^{\mathrm{T}}, \mathbf{v}_{k}^{2}=\left\{\begin{array}{lll}v^{21} & v^{22} & v^{23}\end{array}\right\}^{\mathrm{T}}, \mathbf{v}_{k}^{3}=\left\{\begin{array}{ll}v^{31} & v^{32} v^{33}\end{array}\right\}^{\mathrm{T}}$, the components of the nodal position vector as $\mathbf{x}_{k}=\left\{\begin{array}{llll}x^{1} & x^{2} & x^{3}\end{array}\right\}^{\mathrm{T}}$-dropping the configurations indices $(n)$ and $(n+1)$ it 
is possible to formulate explicit expressions for the linear part of the convective components of the Green-Lagrange strain tensor (Equation (17b)). These expressions are detailed in the appendix, and can be grouped in an equivalent (general) single equation in the form

$$
\mathbf{E}^{u}=\mathbf{M}^{u}(\boldsymbol{\xi})\left\{\begin{array}{l}
\mathbf{d}_{1} \\
\cdots \\
\mathbf{d}_{k}
\end{array}\right\} \quad \text { for } k=1, \ldots, n \text { node and with } n \text { node }=4
$$

leading to the linear convective strain-displacement differential matrix $\left(\mathbf{M}^{u}\right)$ and, afterwards, to the linear displacement-based stiffness matrix. An analogous nodal discretization also applies to the non-linear term in Equation (17b), leading to the corresponding non-linear straindisplacement matrix and, subsequently, the geometric (initial stress) displacement-based stiffness operator.

Due to the non-orthogonal character of the convective frame, the linear strain-displacement operator coming from Equation (17b) will now be projected onto a new orthonormal frame $\left(\mathbf{r}^{1}, \mathbf{r}^{2}, \mathbf{r}^{3}\right)$. This local co-ordinate system is used for the constitutive update, invoking the planestress hypothesis. It is defined at each Gauss point over the undefined mesh (being subsequently updated as deformation occurs).

There is not an unique way to choose the starting configuration for the local frame, and straightforward algorithms, based on the geometry of each finite element in the mesh, can be found in References [37,39]. For the general case of anisotropic elasto-plastic behaviours, however, axes $\mathbf{r}^{1}$ and $\mathbf{r}^{2}$ can be initially taken coincident with the planar anisotropic axes (rolling and transverse direction, respectively), thus forcing $\mathbf{r}^{3}$ to reproduce the normal direction vector. This was the procedure adopted in the solution of the anisotropic elasto-plastic problems in the end of the paper.

After being chosen in the beginning of the simulation, the local frame for each Gauss point is subjected to the rigid-body part of the relative deformation gradient, between configurations $(n)$ and $(n+1)$. In fact, applying a polar decomposition on the relative deformation gradient of Equation (16), it follows that

$$
{ }_{n}^{n+1} \mathbf{F}={ }_{n}^{n+1} \mathbf{R}={ }_{n}^{n+1} \mathbf{U}
$$

where $\left({ }_{n}^{n+1} \mathbf{R}\right)$ is the orthogonal relative rotation tensor and $\left({ }_{n}^{n+1} \mathbf{U}\right)$ corresponds to the relative right stretch tensor. The decomposition in (20) was carried out by means of the algorithm introduced by Franca [48]. Once knowing the local co-ordinate system at stage $(n)$ and the relative rotation tensor between stages $(n)$ and $(n+1)$, it is possible to perform the final update

$$
\left[{ }^{n+1} \mathbf{r}^{1}{ }^{n+1} \mathbf{r}^{2}{ }^{n+1} \mathbf{r}^{3}\right]={ }_{n}^{n+1} \mathbf{R}\left[\begin{array}{llll}
{ }^{n} \mathbf{r}^{1} & { }^{n} \mathbf{r}^{2} & { }^{n} \mathbf{r}^{3}
\end{array}\right]
$$

The same procedure is adopted in the mid-point configuration update of the local frame, from stage $(n)$ up to $\left(n+\frac{1}{2}\right)$, and evaluated from the relative deformation gradient $\left({ }_{n}^{n+1 / 2} \mathbf{F}\right)$.

In doing so, the local co-ordinate system for a given point is then only affected by the rigid body component of the total deformation, characterizing this frame as a 'co-rotational' one, with material strain (and stress) tensors being rotated as if frozen into the deformed continuum [49-54]. As a consequence, the stress update procedure, in the presence of large elasto-plastic deformations, becomes a direct extension of linear small deformation cases, as recently explored by Masud and co-workers $[55,56]$. 
The strain tensor components in the local frame can be obtained, from the convective strain tensor (17), simply by means of the transformation matrix from convective to local frames $\left({ }_{c}^{l} \mathbf{T}\right)$, in the form

$$
{ }_{n}^{n+1} \overline{\mathbf{E}}^{u}={ }_{n}^{n+1} \overline{\mathbf{E}}^{u}\left(\mathbf{r}^{1}, \mathbf{r}^{2}, \mathbf{r}^{3}\right)={ }_{c}^{l} \mathbf{T}_{n}^{n+1} \mathbf{E}^{u}
$$

with the transformation components coming directly from the Jacobian operator between the two reference systems [24,27].

The non-linear (geometric or initial stress) strain-displacement matrix, on the other hand, can be directly referred to the updated local frame in the form [37]

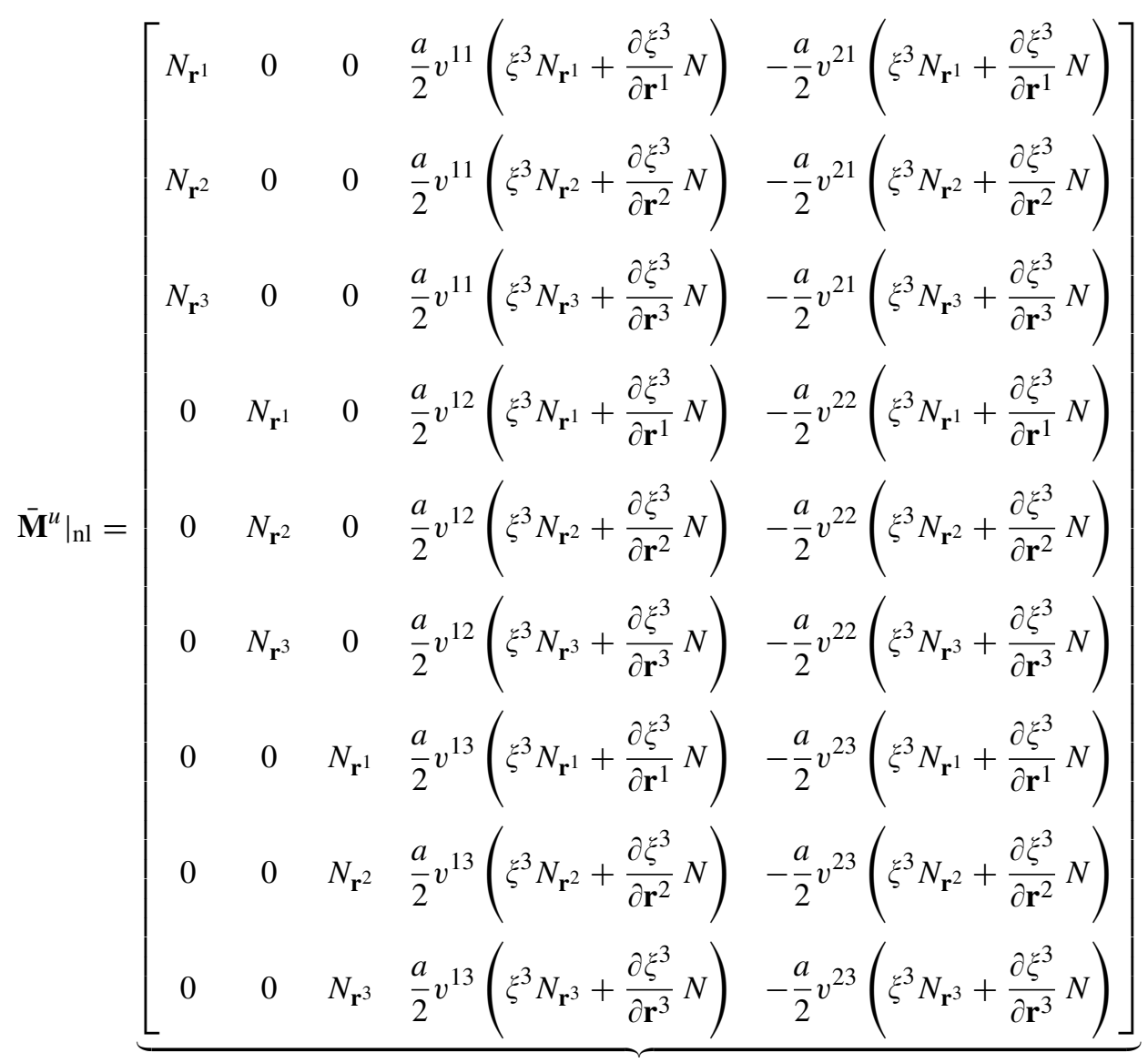

where the subscript (nl) refers to its non-linear character and

$$
N_{\mathbf{r}^{j}}=\frac{\partial N}{\partial \xi^{i}} \frac{\partial \xi^{i}}{\partial \mathbf{r}^{j}}, \quad \text { summation on } i
$$

The basic block presented in Equation (23) is repeated for each node $(k)$ of an element, leading to a matrix $\left.\overline{\mathbf{M}}^{u}\right|_{\mathrm{nl}}$, for the present bilinear (four nodes) shell element, with a total of $(9 \times 20)$ components. 
After detailing the strain field coming from an entirely displacement based formulation, it is time to define the enhanced strain field that will be simply added to the linear strain tensor in (17), in order to eliminate the transverse shear locking and, simultaneously, improve the in-plane behaviour of the original degenerated shell element.

\section{ENHANCED STRAIN FIELD}

\subsection{General aspects}

The core point of this paper relies on the EAS method, in its linear version as originally presented by Simo and Rifai [24]. The main goal in adopting this kind of mixed formulation is, for the present work, the efficient elimination of the transverse shear locking in thin shell structures. The enhanced terms are judiciously chosen and directly included into the convective transverse shear strain field, in order to enlarge the subspace of null (transverse shear strain) energy modes and, consequently, retain the Kirchhoff-Koiter conditions in situations where a pure displacement-based formulation would fail. In this sense, the following analysis relies on the linear shell formulation presented in detail by the authors in Reference [29], although the finite element formulated here is more simple than those introduced in the last reference.

In the present work, and although dealing with non-linearities, the original frame of additive enhancement over the displacement-based convective Green-Lagrange strain tensor is kept, in a way successfully employed at first by Ramm and co-workers (Reference [27] —linear cases and Reference [57] - non-linear cases), and after that by Klinkel et al. [58, 59] and, more recently, consolidated by Vu-Quoc and Tan [60]. As shown in these references, this approach is indeed computationally simpler (and leading to virtually the same results) than the one originally advocated by Simo and Armero [25] and subsequently used, for example, by Simo et al. [26] and Miehe [61].

The total strain field in the EAS method, following its initial formulation, is then assumed to be composed of a compatible (displacement-based) part, coming from Equation (17), and an incompatible (element-wise) part, in the form [24]

$$
\mathbf{E}=\mathbf{E}^{u}+\mathbf{E}^{\alpha}
$$

where the left indexes relating to the configuration were omitted for the sake of simplicity, while the right indexes report to the characteristic of the strain field (displacement $u$ or enhanced internal variables $\alpha$ ). Imposition of the orthogonality condition between stress and enhanced strain field [24-26]

$$
\int_{\mathbf{v}} \mathbf{S}: \mathbf{E}^{\alpha} \mathrm{d} \mathbb{V}=0
$$

reduces the number of independent variables in the Hu-Washizu-Veubeke functional to just two. The weak form of this modified (enhanced) functional is obtained with a Gateaux derivative, leading to the variations of internal and external potentials [60]

$$
\delta \Pi\left(\mathbf{u}, \mathbf{E}^{\alpha}\right)=\delta \Pi^{\mathrm{int}}-\delta \Pi^{\mathrm{ext}}
$$




$$
\begin{aligned}
\delta \Pi^{\mathrm{int}} & =\int_{\mathbf{v}}\left(\delta \mathbf{E}^{u}+\delta \mathbf{E}^{\alpha}\right): \frac{\partial W_{s}\left(\mathbf{E}^{u}+\mathbf{E}^{\alpha}\right)}{\partial\left(\mathbf{E}^{u}+\mathbf{E}^{\alpha}\right)} \mathrm{d} \mathbb{V} \\
\delta \Pi^{\mathrm{ext}} & =\int_{\mathbf{v}} \delta \mathbf{u} \cdot \hat{\mathbf{b}} \rho \mathrm{d} \mathbb{V}+\int_{\mathbf{s}} \delta \mathbf{u} \cdot \hat{\mathbf{t}} \mathrm{d} \mathbb{S}
\end{aligned}
$$

The weak form can now be expanded via a truncated Taylor series about the solution (fixed point) at the $n$th state $\left(\left.\mathbf{u}\right|_{n},\left.\mathbf{E}^{\alpha}\right|_{n}\right)$ [57]

$$
\delta \Pi\left(\left.\mathbf{u}\right|_{n+1},\left.\mathbf{E}^{\alpha}\right|_{n+1}\right) \approx \delta \Pi\left(\left.\mathbf{u}\right|_{n},\left.\mathbf{E}^{\alpha}\right|_{n}\right)+\mathscr{D}[\delta \Pi]\left(\left.\mathbf{u}\right|_{n},\left.\mathbf{E}^{\alpha}\right|_{n}\right) \cdot\left(\Delta \mathbf{u}, \Delta \mathbf{E}^{\alpha}\right)
$$

where, in the present context, the $(\Delta)$ operator relates to a finite variation between $(n)$ and $(n+1)$ states. The finite element interpolation for the enhanced strain field is described next, along with the explicit expression for the $\mathscr{D}[\delta \Pi]$ operator and the main advantages of including the additive approach as in (25).

\subsection{Implementation aspects}

In each element's domain, the displacement field (from Equation (18), with $k=1, \ldots, n$ node, for $n$ node $=4$ ) and its corresponding variation and increment are interpolated in the form

$$
\mathbf{u} \approx \mathbf{u}^{h}=\mathbf{N}(\xi) \mathbf{d}, \quad \delta \mathbf{u} \approx \delta \mathbf{u}^{h}=\mathbf{N}(\xi) \delta \mathbf{d}, \quad \Delta \mathbf{u} \approx \Delta \mathbf{u}^{h}=\mathbf{N}(\xi) \Delta \mathbf{d}
$$

involving the shape functions matrix $\mathbf{N}$ firstly referred to in Equation (2). From the convective expressions for the displacement-based Green-Lagrange strain tensor (Equations (42) and (42)) (see the Appendix Section), the corresponding linear strain-displacement differential operator can be represented as

$$
\mathbf{E}^{u}=\mathbf{M}^{u}(\xi) \mathbf{d}, \quad \delta \mathbf{E}^{u}=\mathbf{M}^{u}(\xi) \delta \mathbf{d}, \quad \Delta \mathbf{E}^{u}=\mathbf{M}^{u}(\xi) \Delta \mathbf{d}
$$

From that exposed in the last section, matrix $\left(\mathbf{M}^{u}\right)$ in Equation (19) is transformed to its local frame counterpart $\left(\overline{\mathbf{M}}^{u}\right)$ being, after that, ready to be used within the implemented constitutive formulation.

In the very same way, the strain-displacement matrix based on the enhanced (internal) variables within an element must be defined. This is carried out, following the original proposal of Simo and Rifai [24], again in the convective frame with the corresponding enhanced functions depending on $(\xi, \eta)$ in the form

$$
\begin{gathered}
\mathbf{E}^{\alpha+\beta}=\mathbf{M}^{\alpha}\left(\xi^{1}, \xi^{2}\right) \boldsymbol{\alpha}_{e}+\mathbf{M}^{\beta}\left(\xi^{1}, \xi^{2}\right) \boldsymbol{\beta}_{e} \\
\delta \mathbf{E}^{\alpha+\beta}=\mathbf{M}^{\alpha}\left(\xi^{1}, \xi^{2}\right) \delta \boldsymbol{\alpha}_{e}+\mathbf{M}^{\beta}\left(\xi^{1}, \xi^{2}\right) \delta \boldsymbol{\beta}_{e} \\
\Delta \mathbf{E}^{\alpha+\beta}=\mathbf{M}^{\alpha}\left(\xi^{1}, \xi^{2}\right) \Delta \boldsymbol{\alpha}_{e}+\mathbf{M}^{\beta}\left(\xi^{1}, \xi^{2}\right) \Delta \boldsymbol{\beta}_{e}
\end{gathered}
$$

It is implicit from this equation that the general enhanced strain tensor $\left(\mathbf{E}^{\alpha}\right)$ in the previous section appears now decomposed into two independent components.

The first part, associated with the strain-displacement matrix $\left(\mathbf{M}^{\alpha}\left(\xi^{1}, \xi^{2}\right)\right)$, is added to the displacement-based transverse shear strain components (modifying the total out-of-plane strain 
energy and, in this way, being responsible for the elimination of transverses shear locking effects). The second part, characterized by $\left(\mathbf{M}^{\beta}\left(\xi^{1}, \xi^{2}\right)\right)$, acts over the displacement based inplane strain energy components, improving the performance of the shell element in bending and membrane-dominated situations. Each of these differential operators involves a distinct number of (element-wise) internal variables, grouped in the internal (non-physical) degrees-of-freedom vectors $\left(\boldsymbol{\alpha}_{e}\right)$ and $\left(\boldsymbol{\beta}_{e}\right)$, respectively.

The first matrix $\left(\mathbf{M}^{\alpha}\left(\xi^{1}, \xi^{2}\right)\right)$ was specifically designed in order to provide an enlargement of the null transverse shear strain subspace for the original bilinear degenerated shell element. A formulation simply based on the displacement field leads to a definite number of components on this subspace basis, which is not enough to ensure null values of the out-of-plane strain energy

$$
\begin{aligned}
& \int_{\square} E_{\xi^{1} \xi^{3}}^{u} \mathrm{~d} \xi^{1} \mathrm{~d} \xi^{2}=0 \\
& \int_{\square} E_{\xi^{2} \xi^{3}}^{u} \mathrm{~d} \xi^{1} \mathrm{~d} \xi^{2}=0
\end{aligned}
$$

at the element level and area $\left(\square \equiv \mathrm{d} \xi^{1} \times \mathrm{d} \xi^{2}\right)$, for limiting low thickness values, as demonstrated by the authors in Reference [29].

Comparative analyses (focusing in detail the null transverse shear strain subspace) between the basic degenerated shell element, bilinear shell elements including assumed strain fields $[7,17-23]$ and also bilinear shell elements based on the selective reduced integration of the transverse shear strain terms [16,4] were performed by the authors [29] within a linear elastic formulation. The main conclusion was that, in order to correct the degenerated formulation from the transverse shear locking point of view, the inclusion of an enhanced transverse strain field is only necessary in the form

$$
\left\{\begin{array}{c}
E_{\xi^{1} \xi^{3}}^{\alpha} \\
E_{\xi^{2} \xi^{3}}^{\alpha}
\end{array}\right\}=\mathbf{M}^{\alpha}\left(\xi^{1}, \xi^{2}\right) \boldsymbol{\alpha}_{e}
$$

where

$$
\begin{aligned}
M_{11}^{\alpha} & =\frac{\partial N_{\alpha}}{\partial \xi^{2}}, \quad M_{12}^{\alpha}=0, \quad M_{13}^{\alpha}=\frac{\partial N_{\alpha}}{\partial \xi^{1}}, \quad M_{14}^{\alpha}=0, \quad M_{15}^{\alpha}=\frac{\partial N_{\alpha}}{\partial \xi^{1}} \frac{\partial N_{\alpha}}{\partial \xi^{2}}, \quad M_{16}^{\alpha}=0 \\
M_{21}^{\alpha} & =0, \quad M_{22}^{\alpha}=\frac{\partial N_{\alpha}}{\partial \xi^{1}}, \quad M_{23}^{\alpha}=0, \quad M_{24}^{\alpha}=\frac{\partial N_{\alpha}}{\partial \xi^{2}}, \quad M_{25}^{\alpha}=0, \quad M_{26}^{\alpha}=\frac{\partial N_{\alpha}}{\partial \xi^{1}} \frac{\partial N_{\alpha}}{\partial \xi^{2}} \\
\boldsymbol{\alpha}_{e} & =\left\{\begin{array}{llllll}
\alpha_{1} & \alpha_{2} & \alpha_{3} & \alpha_{4} & \alpha_{5} & \alpha_{6}
\end{array}\right\}^{\mathrm{T}}
\end{aligned}
$$

and accounting for a bubble shape function in the form $\left(N_{\alpha}=\left(1-\xi^{1} \xi^{1}\right)\left(1-\xi^{2} \xi^{2}\right)\right)$.

At this point it is worth noting that the preceding analysis was entirely based on a fully numerically integrated formulation (corresponding, for the bilinear four node shell element, to a $2 \times 2$ integration rule). This integration quadrature is also strictly employed in the strain field. It is interesting to note that, starting with an a priori complete in-plane integration 
for the displacement strain field-as stated before-the enhanced strain field necessary to the elimination of transverse shear locking effects turned out to retain a kind of reduced integrated character, coming from the quadratic terms in the bubble function. In this sense, this formulation is in contrast to some works in the literature, sometimes resorting to higher quadrature rules specifically for the enhanced strain field, in order to improve the performance and remove instabilities from the elements (as in References [26,62,63], just to name some classical works).

Additionally, from the analysis of Equations (33), comes that no dependence on the $\left(\xi^{3}\right)$ orientation is introduced into the enhanced strain field. This turns the contribution of the enhanced transverse shear strain terms invariant respecting the number of integration points along thickness direction, which is clear an advantage for numerical integration procedures in elasto-plastic problems.

After detailing the relevant aspects of the first enhanced interpolation matrix $\left(\mathbf{M}^{\alpha}\right)$, it is necessary to establish the topology of the enhanced matrix $\left(\mathbf{M}^{\beta}\right)$ in (31). This term is intended to affect only the in-plane strain field, improving the element performance in membranedominated situations and being decoupled from the transverse shear strain enhancement. The choice of the in-plane EAS field, proposed by Andelfinger and Ramm [27] and involving seven internal variables per element, gave rise to the linear research introduced in Reference [29]. In the present work, however, a distinct enhanced strain field is adopted involving just five enhanced variables per element, with no loss in accuracy when compared to the previous formulation. In fact, the numerical results for both in-plane enhancements proved to lead to similar results in a series of benchmark tests, with the choice of five internal variables seeming to be an optimal choice for reliable results, as detailed in the report [33].

Doing so, the membrane/bending EAS modes are given by the following interpolation:

where

$$
\left\{\begin{array}{c}
E_{\xi^{1} \xi^{1}}^{\beta} \\
E_{\xi^{2} \xi^{2}}^{\beta} \\
E_{\xi^{1} \xi^{2}}^{\beta}
\end{array}\right\}=\mathbf{M}^{\beta}\left(\xi^{1}, \xi^{2}\right) \boldsymbol{\beta}_{e}
$$

$$
\begin{array}{lllll}
M_{11}^{\beta}=\xi^{1}, & M_{12}^{\beta}=0, & M_{13}^{\beta}=0, & M_{14}^{\beta}=0, & M_{15}^{\beta}=0 \\
M_{21}^{\beta}=0, & M_{22}^{\beta}=\xi^{2}, \quad M_{23}^{\beta}=0, & M_{24}^{\beta}=0, & M_{25}^{\beta}=0 \\
M_{31}^{\beta}=0, & M_{32}^{\beta}=0, & M_{33}^{\beta}=\xi^{1}, & M_{34}^{\beta}=\xi^{2}, & M_{35}^{\beta}=\xi^{1} \xi^{2} \\
\boldsymbol{\beta}_{e}=\left\{\begin{array}{lllll}
\beta_{1} & \beta_{2} & \beta_{3} & \beta_{4} & \beta_{5}
\end{array}\right\}^{\mathrm{T}}
\end{array}
$$

The in-plane EAS interpolation matrix (34), in conjunction to the out-of-plane (transverse shear) in Equations (33), define the formulation of the present S4E6P5 shell element. The acronym comes from the characterization of a shell element with four nodes, six transverse shear enhanced variables, plus five in-plane enhanced variables. This lower number of internal variables automatically influences the computational costs involved in a given analysis, mainly when considering elasto-plastic material modelling, as within the scope of the present paper. 


\section{ALGORITHMIC TREATMENT OF NON-LINEARITIES}

\subsection{Geometric non-linearity}

After the description of the interpolation functions and variables for both the displacementbased and enhanced strain fields (in-plane and out-of-plane strain fields), the second member of the right-hand side of the linearized weak form (28) can be rewritten (dropping the state and elemental indices) as [57-60]

$$
\mathscr{D}[\delta \Pi](\mathbf{d}, \boldsymbol{\alpha}, \boldsymbol{\beta}) \cdot(\Delta \mathbf{d}, \Delta \boldsymbol{\alpha}, \Delta \boldsymbol{\beta})=\frac{\partial\left(\delta \Pi^{\mathrm{int}}-\delta \Pi^{\mathrm{ext}}\right)}{\partial(\mathbf{d}, \boldsymbol{\alpha}, \boldsymbol{\beta})} \cdot(\Delta \mathbf{d}, \Delta \boldsymbol{\alpha}, \Delta \boldsymbol{\beta})
$$

Including the corresponding displacement and enhanced based interpolation, the variations in (35) take the matricial form

$$
\begin{aligned}
\delta \Pi^{\mathrm{int}}(\mathbf{d}, \boldsymbol{\alpha}, \boldsymbol{\beta}) & =\delta \mathbf{d}^{\mathrm{T}} \int_{\mathbb{V}}\left(\overline{\mathbf{M}}^{u}\right)^{\mathrm{T}} \overline{\mathbf{S}} \mathrm{d} \mathbb{V}+\delta \boldsymbol{\alpha}^{\mathrm{T}} \int_{\mathbb{V}}\left(\overline{\mathbf{M}}^{\alpha}\right)^{\mathrm{T}} \overline{\mathbf{S}} \mathrm{d} \mathbb{V}+\delta \boldsymbol{\beta}^{\mathrm{T}} \int_{\mathbb{V}}\left(\overline{\mathbf{M}}^{\beta}\right)^{\mathrm{T}} \overline{\mathbf{S}} \mathrm{d} \mathbb{V} \\
\delta \Pi^{\mathrm{ext}}(\mathbf{d}) & =\delta \mathbf{d}^{\mathrm{T}} \int_{\mathbb{V}} \mathbf{N}^{\mathrm{T}} \hat{\mathbf{b}} \rho \mathrm{d} \mathbb{V}+\delta \mathbf{d}^{\mathrm{T}} \int_{\mathbb{S}} \mathbf{N}^{\mathrm{T}} \hat{\mathbf{t}} \mathrm{d} \mathbb{S}
\end{aligned}
$$

where the $\left(\overline{\mathbf{M}}^{\alpha}\right)$ and $\left(\overline{\mathbf{M}}^{\beta}\right)$ matrices refer to the enhanced strain operator in the local frame while, in the same way, $(\overline{\mathbf{S}})$ points to the second Piola-Kirchhoff stress tensor also related to the local orthogonal frame.

Focusing on the variation of the internal part (36a) of the whole potential, it is possible to state that

$$
\begin{aligned}
\mathscr{D}\left[\delta \Pi^{\mathrm{int}}\right] \cdot(\Delta \mathbf{d}, \Delta \boldsymbol{\alpha}, \Delta \boldsymbol{\beta})= & \frac{\partial\left(\delta \Pi^{\mathrm{int}}\right)}{\partial \mathbf{d}} \cdot \Delta \mathbf{d}+\frac{\partial\left(\delta \Pi^{\mathrm{int}}\right)}{\delta \boldsymbol{\alpha}} \cdot \Delta \boldsymbol{\alpha}+\frac{\partial\left(\delta \Pi^{\mathrm{int}}\right)}{\partial \boldsymbol{\beta}} \cdot \Delta \boldsymbol{\beta} \\
= & \delta \mathbf{d}^{\mathrm{T}}\left[\left(\mathbf{K}_{u u}^{l g}+\mathbf{K}_{u u}^{n l g}\right) \Delta \mathbf{d}+\mathbf{K}_{u \alpha} \Delta \boldsymbol{\alpha}+\mathbf{K}_{u \beta} \Delta \boldsymbol{\beta}\right] \\
& +\delta \boldsymbol{\alpha}^{\mathrm{T}}\left[\mathbf{K}_{\alpha u} \Delta \mathbf{d}+\mathbf{K}_{\alpha \boldsymbol{\alpha}} \Delta \boldsymbol{\alpha}\right]+\delta \boldsymbol{\beta}^{\mathrm{T}}\left[\mathbf{K}_{\beta u} \Delta \mathbf{d}+\mathbf{K}_{\beta \beta} \Delta \boldsymbol{\beta}\right] \quad(\forall \delta \mathbf{d}, \forall \delta \boldsymbol{\alpha}, \forall \delta \boldsymbol{\beta})
\end{aligned}
$$

Linear and non-linear geometric (initial stress) stiffness matrices $\left(\mathbf{K}_{u u}^{l g}\right.$ and $\mathbf{K}_{u u}^{n l g}$, respectively) are defined as in a fully displacement-based formulation [37], coming from the expressions defined in Section 2.

The main result of the inclusion of the enhanced parameters into the variational formulation is the appearance of the coupling stiffness matrices $\left(\mathbf{K}_{\alpha u}, \mathbf{K}_{u \alpha}\right)$ and $\left(\mathbf{K}_{\beta u}, \mathbf{K}_{u \beta}\right)$, as well as the introduction of the fully enhanced stiffness operators $\left(\mathbf{K}_{\alpha \alpha}\right)$ and $\left(\mathbf{K}_{\beta \beta}\right)$, all of them possessing the same structure as in the linear formulation of Reference [24]. In fact, the adopted additive approach in the way of Equation (25) leads to a straightforward algorithmic extension from the linear case, with no inclusion of non-linear geometric stiffness matrices associated with the enhanced variables, as happens in formulations based on the multiplicative enhancement of the deformation gradient (References [25, 61], among others). 
The result is then an equivalent system of equations, on matrix form, with the structure [57-60]:

$$
\begin{gathered}
{\left[\begin{array}{ccc}
\left(\mathbf{K}_{u u}^{l g}+\mathbf{K}_{u u}^{n l g}\right) & \mathbf{K}_{u \alpha} & \mathbf{K}_{u \beta} \\
\mathbf{K}_{\alpha u} & \mathbf{K}_{\alpha \alpha} & 0 \\
\mathbf{K}_{\beta u} & 0 & \mathbf{K}_{\beta \beta}
\end{array}\right]\left\{\begin{array}{c}
\Delta \mathbf{d} \\
\Delta \boldsymbol{\alpha} \\
\Delta \boldsymbol{\beta}
\end{array}\right\}} \\
=\left\{\begin{array}{c}
\int_{\mathbb{V}} \mathbf{N}^{\mathrm{T}} \overline{\mathbf{b}} \rho \mathrm{d} \mathbb{V}+\int_{\mathbb{S}} \mathbf{N}^{\mathrm{T}} \overline{\mathbf{t}} \mathrm{d} \mathbb{S}-\int_{\mathbb{V}}\left(\overline{\mathbf{M}}^{u}\right)^{\mathrm{T}} \overline{\mathbf{S}} \mathrm{d} \mathbb{V} \\
-\int_{\mathbb{V}}\left(\overline{\mathbf{M}}^{\alpha}\right)^{\mathrm{T}} \overline{\mathbf{S}} \mathrm{d} \mathbb{V} \\
-\int_{\mathbb{V}}\left(\overline{\mathbf{M}}^{\beta}\right)^{\mathrm{T}} \overline{\mathbf{S}} \mathrm{d} \mathbb{V}
\end{array}\right\}
\end{gathered}
$$

The internal force vectors related to displacement and enhanced fields, $\left(\int_{\mathbb{V}}\left(\overline{\mathbf{M}}^{u}\right)^{\mathrm{T}} \overline{\mathbf{S}} \mathrm{d} \mathbb{V}\right.$, $\left.\int_{\mathbb{V}}\left(\overline{\mathbf{M}}^{\alpha}\right)^{\mathrm{T}} \overline{\mathbf{S}} \mathrm{d} \mathbb{V}, \int_{\mathbb{V}}\left(\overline{\mathbf{M}}^{\beta}\right)^{\mathrm{T}} \overline{\mathbf{S}} \mathrm{d} \mathbb{V}\right)$ come from the discrete form of Equation (27b). Also, no coupling between the two enhanced strain field appears in the equivalent expression (38), and the two fields can be treated altogether in the implementation.

\subsection{Material non-linearity}

Although the enhanced strain field is defined based on the (material) (Green-Lagrange local strain tensor, energetically conjugated to the second Piola-Kirchhoff local stress tensor, the stress field to be constitutive updated in the incremental-iterative process is the (spatial) Cauchy stress tensor. This is possible since the incremental stress is referred to the local frame detailed before, updated with the rotation tensor coming from the polar decomposition. In this way, the local orthogonal system is only affected by the rigid-body rotation part of the deformation, acting as a 'co-rotational' reference frame. A detailed description of the algorithmic steps involved can be found in classical works in References [40,64-70].

The last detail in the implementation of the constitutive equations is the transformation of the incremental spatial tensors to incremental material tensors, in order to directly include the displacement and enhanced-based formulations described in Section 2. For small strains $\left({ }_{n}^{n+1} \mathbf{U} \approx \mathbf{I}_{2}\right)$ and following the previously defined rotated local axes, the incremental rotated (co-rotational) Cauchy stress tensor turns to be equivalent to the incremental rotated second Piola-Kirchhoff stress tensor [54,66], and it is possible to write a final incremental rotated stress update equation in the form $[55,56]$

$$
{ }^{n+1} \overline{\boldsymbol{\sigma}}={ }^{n} \overline{\boldsymbol{\sigma}}+{ }_{n}^{n+1} \overline{\boldsymbol{\sigma}}={ }^{n} \overline{\boldsymbol{\sigma}}+{ }_{n}^{n+1} \overline{\mathbf{S}}
$$

Increment in the rotated second Piola-Kirchhoff stress tensor comes from the increment of the rotated (local-based) Green-Lagrange strain field, between $(n)$ and $(n+1)$ configurations, and approximated at a mid-point configuration in the form

$$
{ }_{n}^{n+1} \overline{\mathbf{E}}={ }_{n}^{n+1 / 2} \overline{\mathbf{M}}^{u n+1} \mathbf{d}+{ }_{n}^{n+1 / 2} \overline{\mathbf{M}}^{\alpha}{ }_{n}^{n+1} \boldsymbol{\alpha}+{ }_{n}^{n+1 / 2} \overline{\mathbf{M}}^{\beta}{ }_{n}^{n+1} \boldsymbol{\beta}
$$


Strain-displacement matrices, either for displacement and EAS fields, are constructed upon the definition of the local frame at the mid-point configuration, coming from analogous relations than those presented in Equations (20) and (21).

Related to the elasto-plastic algorithm implementation, a standard 'elastic predictor plastic corrector' radial return algorithm in the sense of References [71, 72] was implemented, properly adapted to account for either isotropic and anisotropic planar and normal yield criteria. Detailed implementation steps can be found in Reference [33], being omitted here for the sake of understandability purposes.

In the following section, large displacement and rotation elasto-plastic problems are presented, in order to assess the reliability of the before described formulation. Isotropic yield criteria in the following refer to the standard $J_{2}$ plasticity model of von Mises, while for anisotropic plasticity the (planar and normal) models of Hill [73], as well as the criterion from Barlat et al. [74], were implemented.

\section{NUMERICAL EXAMPLES}

\subsection{Isotropic elasto-plastic constitutive behaviour}

5.1.1. Channel-section beam with plasticity. A channel-section (U-shaped) beam, clamped at one end and subjected to a concentrated force at its free end, is analysed. The geometry is according to Figure 1, where values for length, width, height and thickness of the beam follow those originally proposed in Reference [75]. Among well-known works following with the treatment of this problem within shell formulations are, for instance, References [76-79]. All of these formulations employ drilling degrees-of-freedom. In the particular case of Reference [77], folded zones of the beam are modelled with rigid intersections, accounting for three rotational degrees-of-freedom. Also for these formulations, only non-linear geometric analyses were performed, except for Reference [78], where for the first time elasto-plasticity were accounted for. Therefore, these results will be taken as the reference ones, for the present work.

An initial yield stress $\sigma_{y 0}=5.0 \times 10^{3}$ and isotropic hardening coefficient $H_{\text {iso }}=0.0$ (perfect plasticity) were adopted. The director update procedure equations (10) and (11) and its inextensibility condition, adopted in the present work, are similar to the procedures adopted in the so-called '5/6 parameter' formulation in Reference [78, pp. 247-248]. The main difference remains in that the present $S 4 E 6 P 5$ shell element does not include drilling rotations in its formulation, treating in the same way smooth and folded thin plates or shells. According to the conclusions of Eberlein and Wriggers [78], such a formulation would 'completely fail in the present example'. However, the behaviour of the proposed enhanced strain element is quite acceptable (mainly in the combined elasto-plastic non-linear geometric case), as can be clearly seen in Figure 2, where the displacement of the loaded node (in the direction of $F$ in Figure 1 ) is shown graphically, both for only geometric (nlgeom) and geometric plus material (nlgeom $+e p$ ) non-linearities. In Reference [78], a mesh consisting of $20 \times 72$ shell elements was considered, while in the present work $20 \times 36$ S4E6P5 elements were adopted (further mesh refinements have given the same results).

Related to the number of load steps in each analysis, the non-linear geometric path was achieved in 56 increments, compared to 90 for the full geometric and material non-linear 


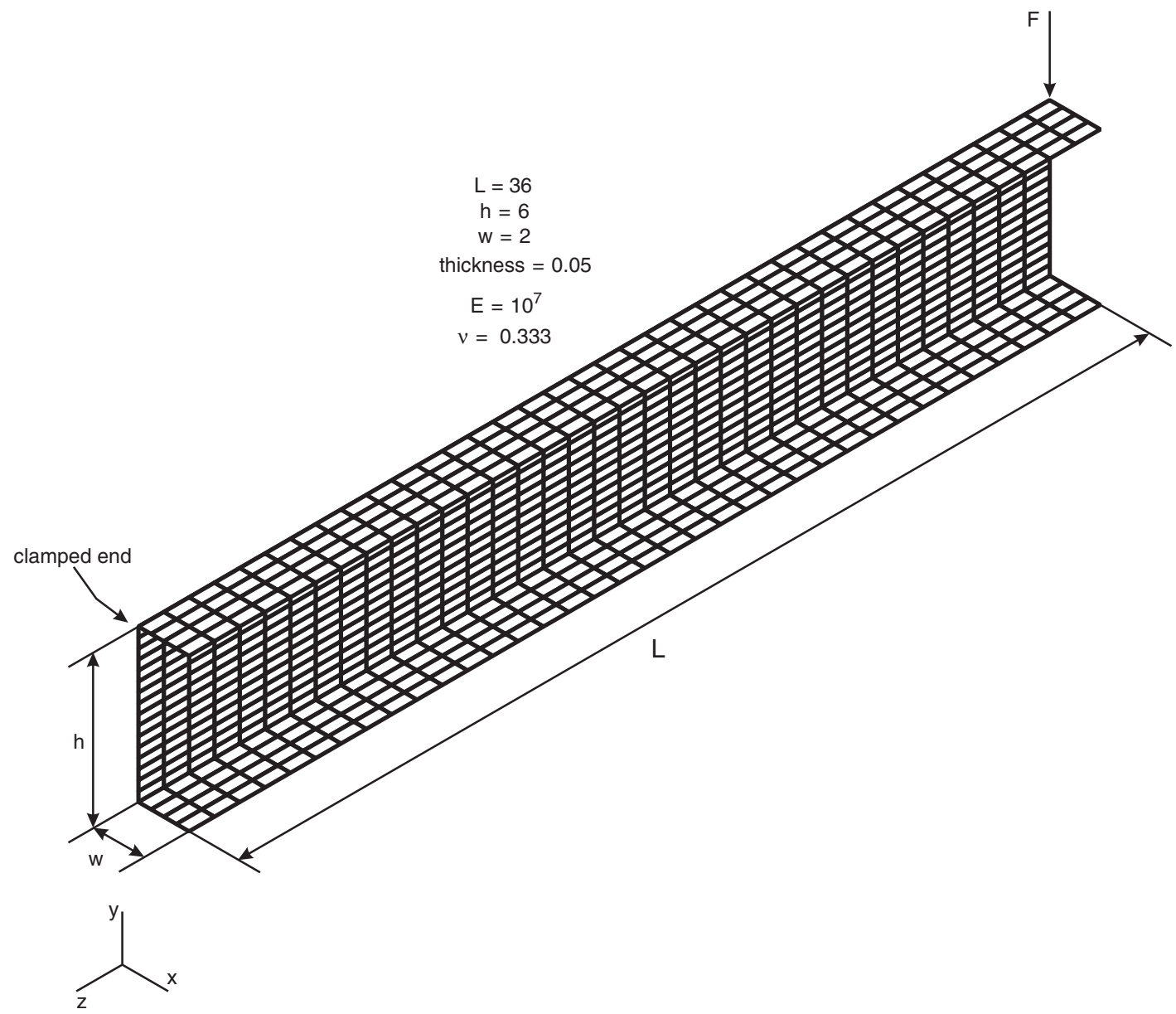

Figure 1. Channel-section beam-model with a mesh of $36 \times 20$ elements, including geometric, boundary and loading conditions.

behaviour. For both cases, the 'cylindrical' arc-length control procedure [80-82] was implemented. It is also worth mention that just two Gauss points along thickness direction were adopted (in opposition to Reference [78], employing five Gauss points along thickness direction).

As stated in Reference [78], equivalent plastic strain peaks occur near the clamped end of the structure, starting in early stages of loading. With the increase of deformation, however, a spread of plastic zones along the longitudinal direction of the beam is verified, as shown in Figure 3, where the deformed configuration for an absolute displacement of 1.471 (of the loaded node point) is represented.

5.1.2. Elasto-plastic analysis of a simply-supported plate. In this example, a simply-supported square plate is subjected to pressure loads, as shown schematically in Figure 4. This example has been treated in a number of references, including shell and solid-shell formulations and 


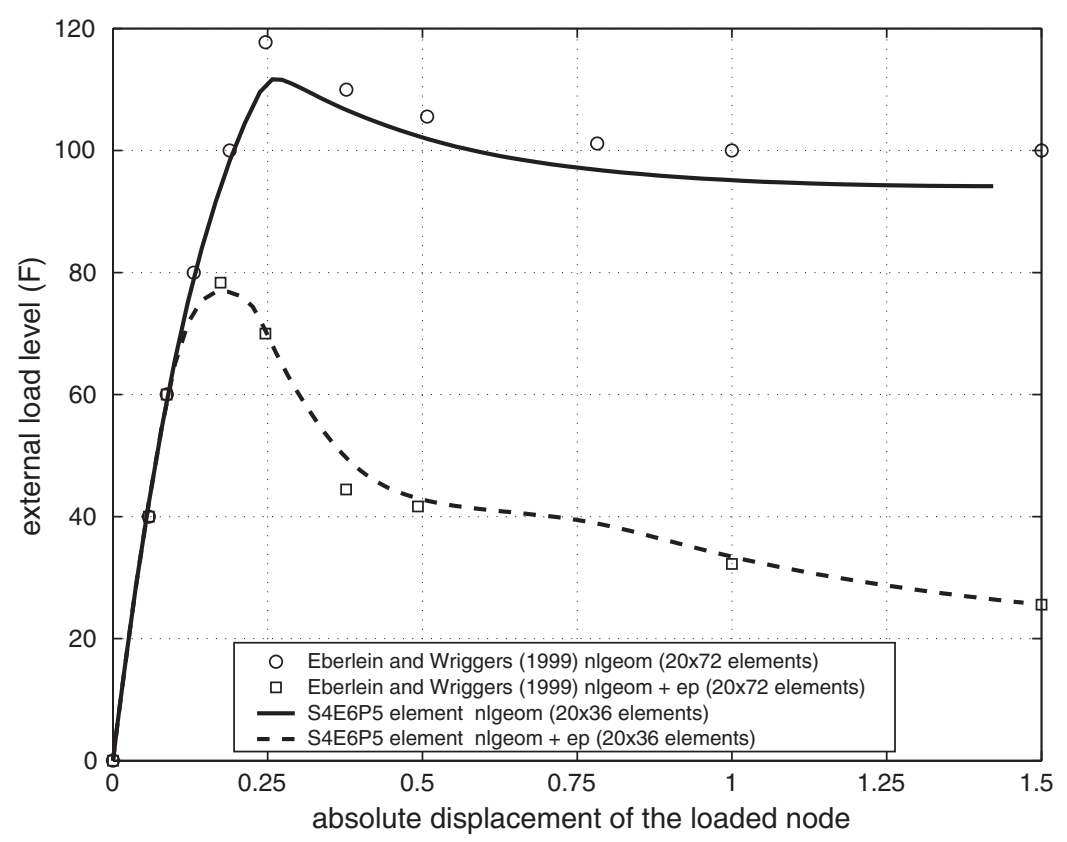

Figure 2. Channel-section beam-deflection curves for the loaded node.

adopting a variety of mesh topologies (see, for instance References [28, 61, 78, 83-86]). About Figure 4, and following the previous references, the total length of the plate is $2 L=508$, with thickness $a=2.54$ consistent unities. Only one quarter of the plate needs to be modelled, due to symmetry. Material behaviour is described by a Young's modulus $E=6.9 \times 10^{4}$, Poisson's ratio $v=0.3$, initial yield stress $\sigma_{y 0}=248$ and isotropic hardening coefficient $H_{\text {iso }}=0.0$ (for a linear hardening law of the type $\sigma_{y}\left(\varepsilon^{p}\right)=\sigma_{y 0}+H_{\text {iso }} \varepsilon^{p}$ ). For each simulation just two Gauss points along thickness direction are employed, as for a higher interpolation order the same results were obtained. For comparison, Reference [78] adopts five Gauss points along thickness direction, while References $[85,86]$ use six integration points. About boundary and loading conditions, displacements along the $O Z$ direction are restrained on the outer edges, while a deformation dependent pressure load $p=f \times p_{0}$ is applied on one side of the shell, for a nominal load level of $p_{0}=10^{-2}$.

In the present work, four mesh topologies were adopted to attest the convergence behaviour of the proposed enhanced strain formulation: a first coarser mesh consisting of equally spaced $15 \times 15$ shell elements (Figure 4); a second mesh with the same number of elements but refined towards the outer corners [78]; a third mesh consisting of $24 \times 24$ regularly spaced elements [83] and a last, extremely refined, mesh with 1375 elements [41]. The last three meshes are schematically represented in Figure 5.

Figure 6 shows the load-deflection curve, for a maximum load factor $(f=60.0)$, with the corresponding out-of-plane deflection of the central point of the plate (point $C$ in Figure 5). Results are compared to those provided in References [78,83]. Within the analysed meshes, all of them converge to the solution presented by Betsch and Stein [83]. The only exception is the regular mesh with $15 \times 15$ shell elements, and just for the range of displacement between 


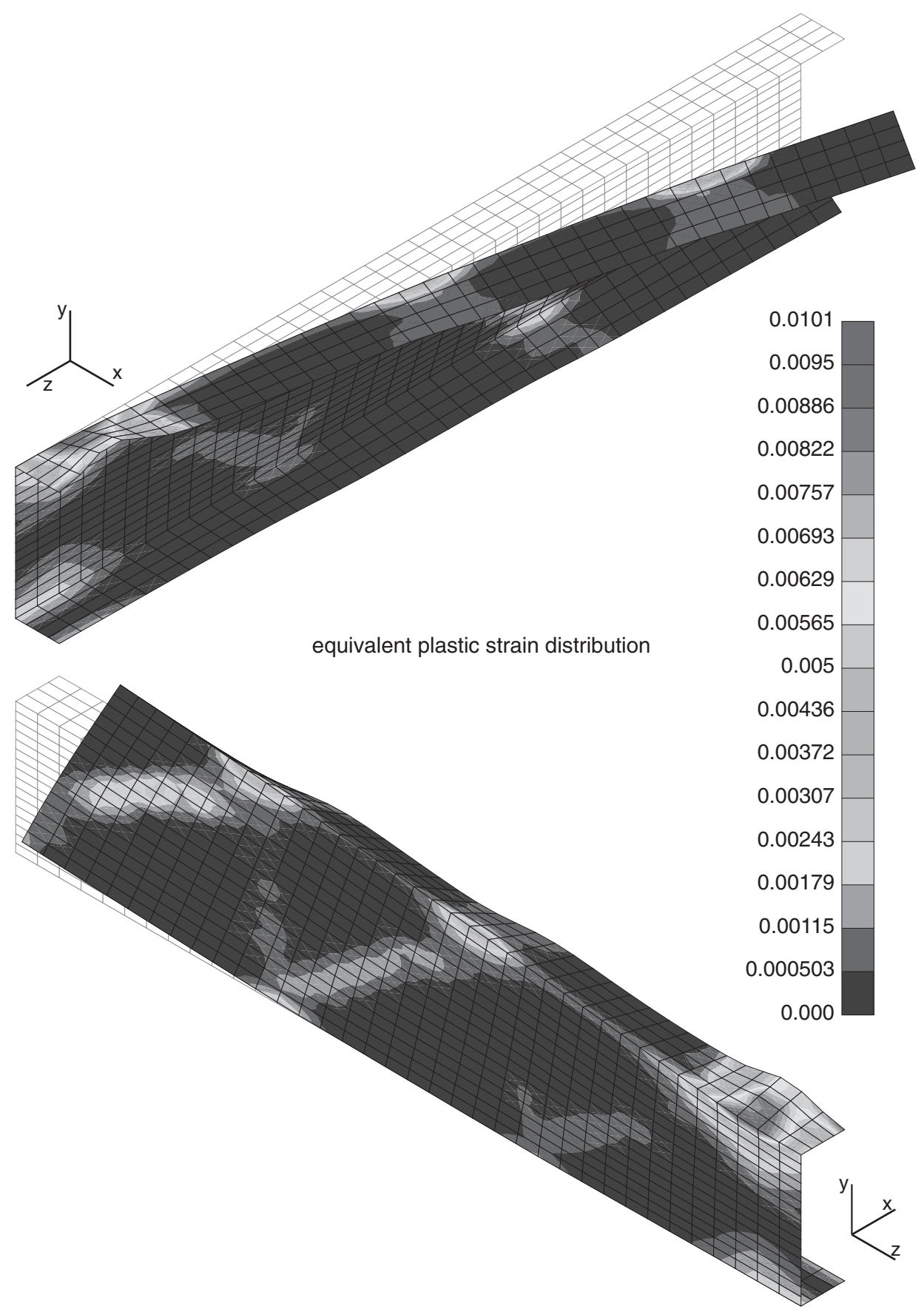

Figure 3. Channel-section beam-deformed configuration and equivalent plastic strain for a displacement value of 1.471 . 


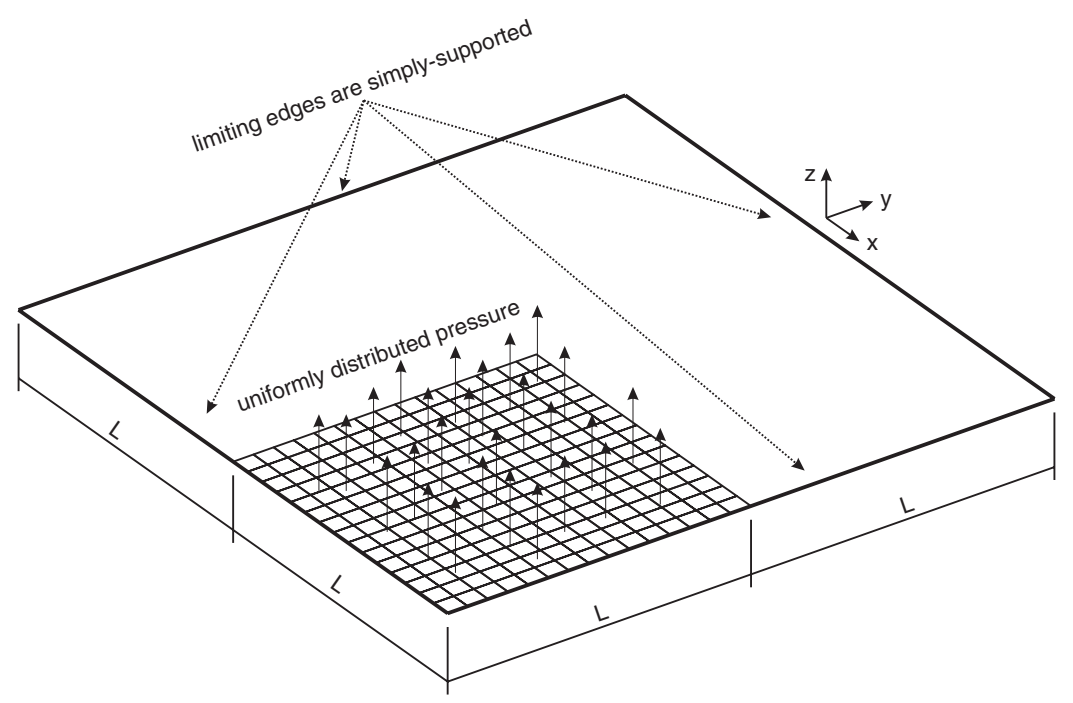

Figure 4. Simply-supported plate-problem definition with a representative regular mesh of $15 \times 15$ elements.

30 and 65 consistent unities, where the present solution approaches the one by Eberlein and Wriggers [78]. However, the overall behaviour obtained with the present S4E6P5 shell element is quite good.

The deformed structures $(f=60.0)$ for each mesh assume a 'pillow-like' configuration, with the elements in the corner zones being subjected to a high level of out-of-plane distortions (more pronounceable with the regular mesh of $15 \times 15$ elements). From the analysis of the equivalent plastic strain levels, it is noticeable the onset of their maximum values in the corner zones of the plates. More importantly, it is possible to assess some level of mesh dependency of the equivalent plastic strain values, an interesting aspect which is not addressed in the previous references. For the present case, the values of the maximum equivalent plastic strain values are as following: $0.190(15 \times 15$ regular mesh $) ; 0.249(15 \times 15$ refined mesh $) ; 0.214(24 \times 24$ regular mesh) and 0.343 (1375 elements' mesh). For the sake of completeness, the number of load increments necessary to reach the load factor $(f=60.0)$ are: 44 for the $15 \times 15$ regular mesh; 47 for the $15 \times 15$ refined mesh; 54 for the $24 \times 24$ mesh and, finally, 56 for the more refined mesh of 1375 elements.

A deeper insight of the structural behaviour can be retained with the increase of the load factor applied to the pressure loads. In Figure 7, six deformation stages of the plate (top view) are represented, for the refined mesh of 1375 elements. The first one (Figure 7(a)) represents the deformed plate for a load factor $(f=285.4)$. At this stage, the out-of-plane displacement verified for point $C$ (Figure 5) is equal to 121.4 consistent unities. Even more relevant is the fact that, at this load factor, it can be verified the onset of wrinkles at the mid-side of each plate's edge. With a further increase of the load factor, this phenomenon is even more noticeable, as represented in the sequence on Figure 7(b) (load factor $f=386.8$ ). Figure 7(c) (load factor $f=479.9$ ) and Figure 7(d) (load factor $f=557.6$ ), corresponding to central point displacements of 131.6, 140.5 and 149.0, respectively. Above this load level, and with an 

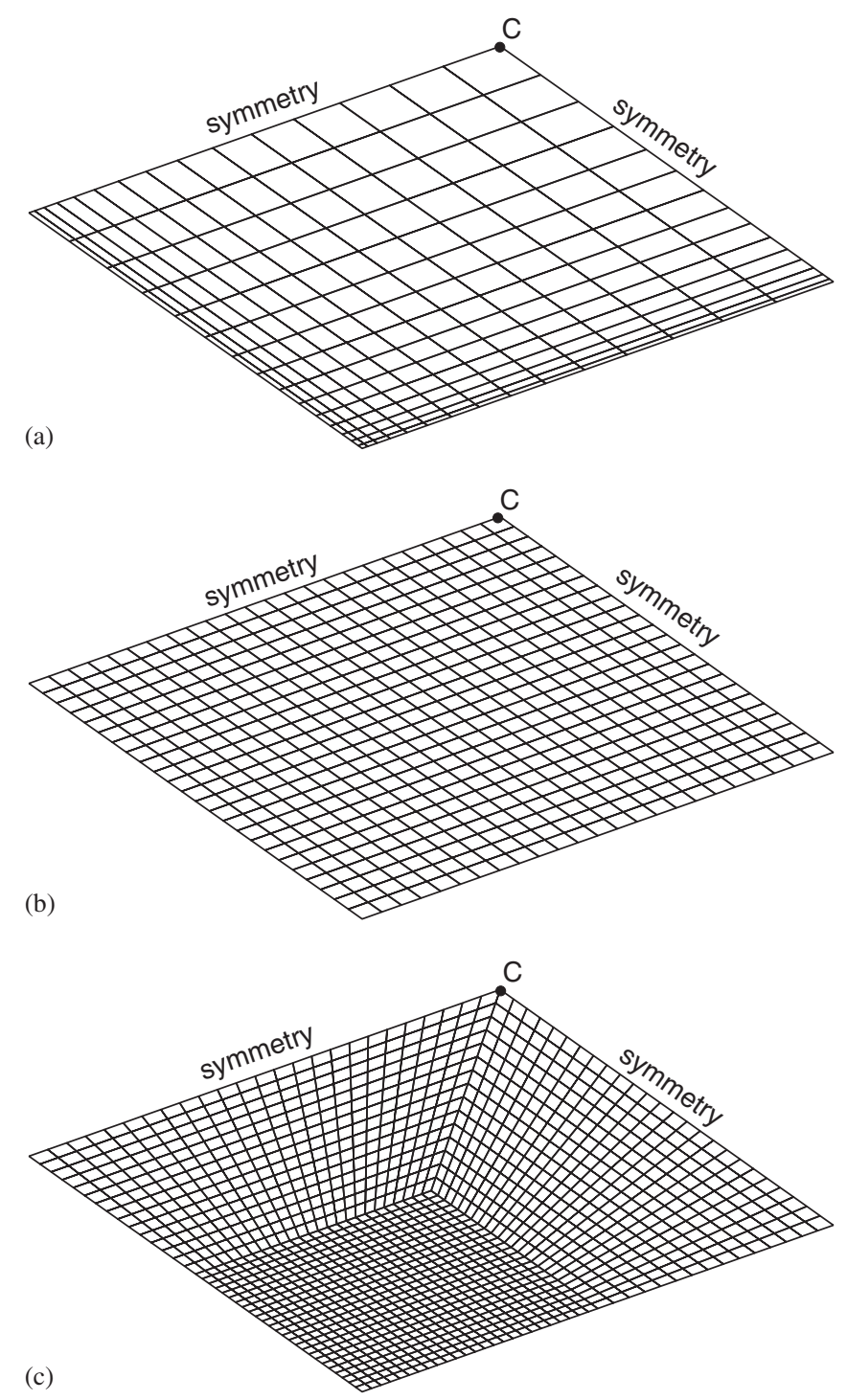

Figure 5. Simply-supported plate-adopted meshes with: (a) $15 \times 15$; (b) $24 \times 24$; and (c) 1375 shell elements.

even higher load factor, the plate is only able to accommodate more deformation if adopting a spherical shape. The corner zones of the plate (still those with higher levels of plastic strains) tend, therefore, to assume a rounded shape (losing their sharp aspect still visible on Figure 7(a)-(c)). The spread of plastic areas is noticeable, starting in configuration (b), with the plate, for the final two steps (Figure 7(e) and (f)), virtually covered by plastic zones as a whole. Displacement of the central point for the last two configurations is equal to 157.0 and 164.5, for load factors of $(f=600.7)$ and $(f=635.5)$, respectively. Dimetric projections 


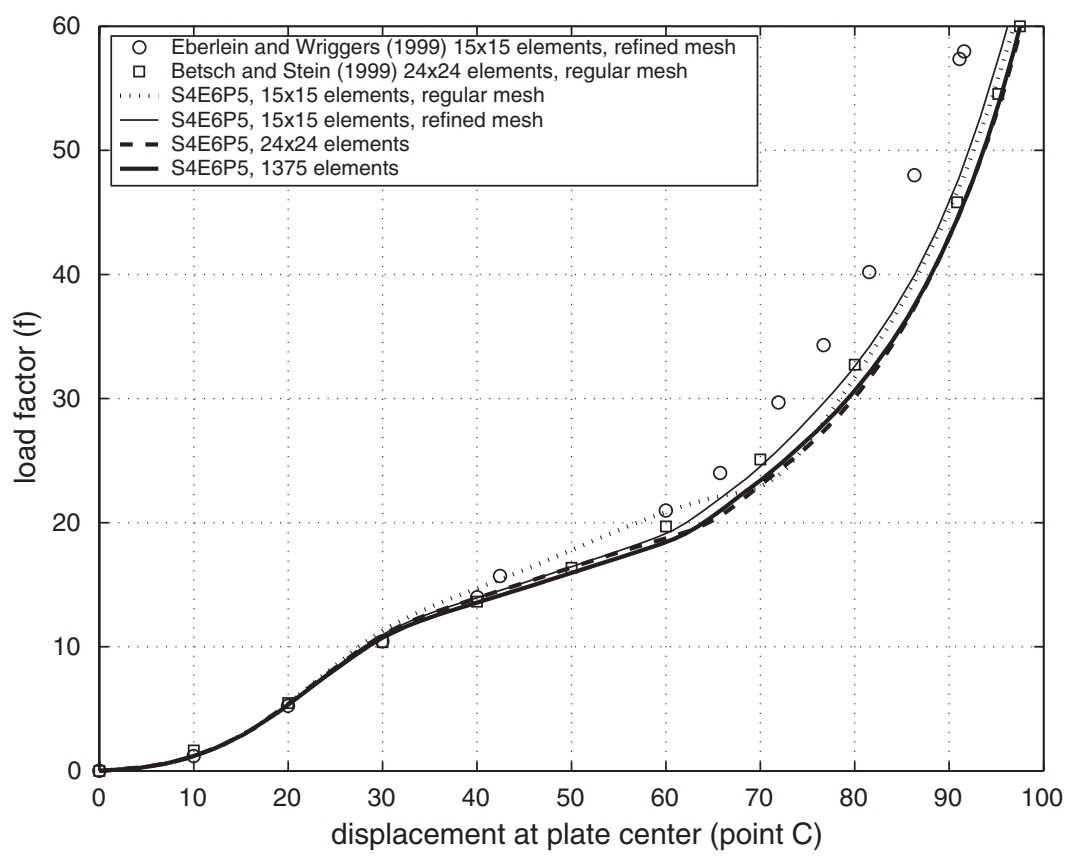

Figure 6. Simply-supported plate-load-deflection curves for a limit load factor $f=60$.

of configurations at load levels (a), (c) and (f) are reproduced in Figure 8, where the final rounded shape of the plate can be further observed.

5.1.3. Pinched cylinder including elasto-plasticity. This example deals with a cylindrical shell, subjected to a pair of concentrated loads and bounded by rigid diaphragms on its extremities. It is a common benchmark in shell and solid-shell elements technology, and have been treated by a number of authors (firstly by Simo and Kennedy [87] and after that in, for instance, References $[61,72,78,88-90])$. In the present work, the problem will be considered in the same way as stated in the last references, particularly following References [61,78].

The initial geometry of the shell is as illustrated in Figure 9, where the mesh area is just one-eighth of the cylinder, due to symmetry reasons. Geometrical data refer to the whole length of the cylinder $L=600$, the radius $R=300$ and thickness $a=3$. The rigid diaphragm boundary condition acts in a way that only displacements along the $O Y$ direction are allowed in the cylinder free-ends. Material properties are the elasticity modulus $E=3000.0$, Poisson's coefficient $v=0.3$, initial yield stress $\sigma_{y 0}=24.3$ and isotropic hardening coefficient $H_{\text {iso }}=$ 300.0 , for the same linear hardening law used in the previous examples.

Following References [61,78], a mesh consisting of $32 \times 32$ S4E6P5 shell elements is employed. Also, an incremental load controlled procedure, based on the Newton-Raphson method with the 'cylindrical' arc-length control, is used. In the set of works cited before, on the other hand, displacement controlled advance methods were employed to grant stable convergence. 

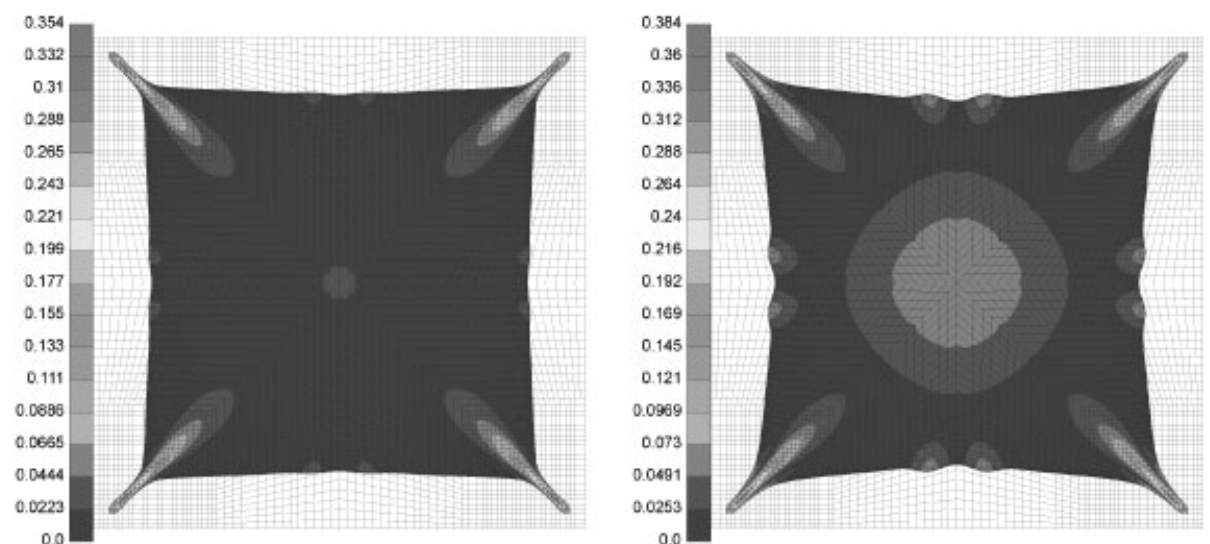

(a)

(b)
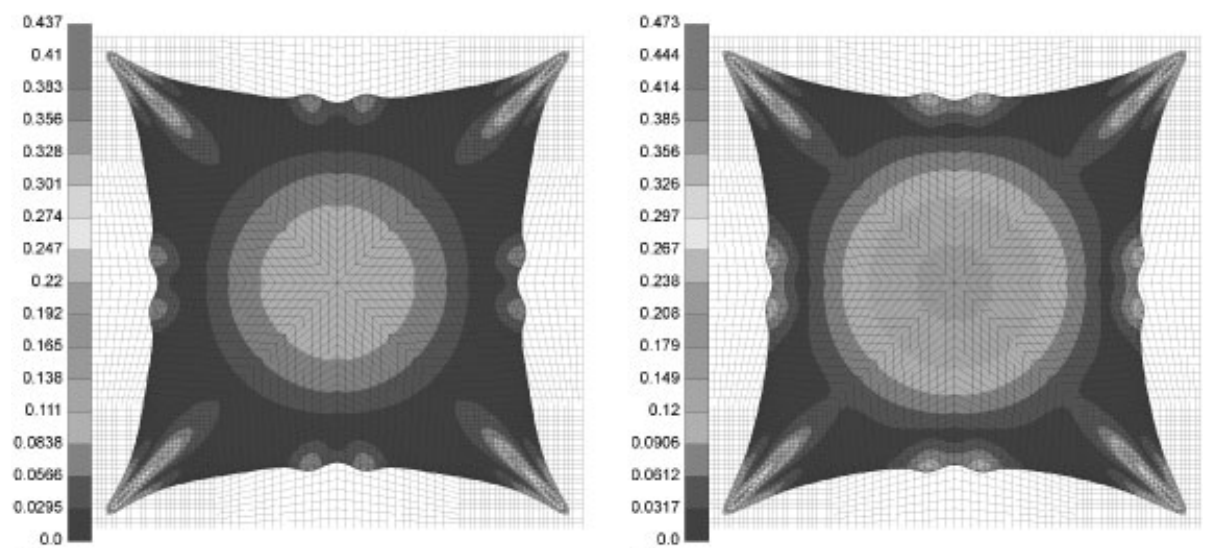

(c)

(d)
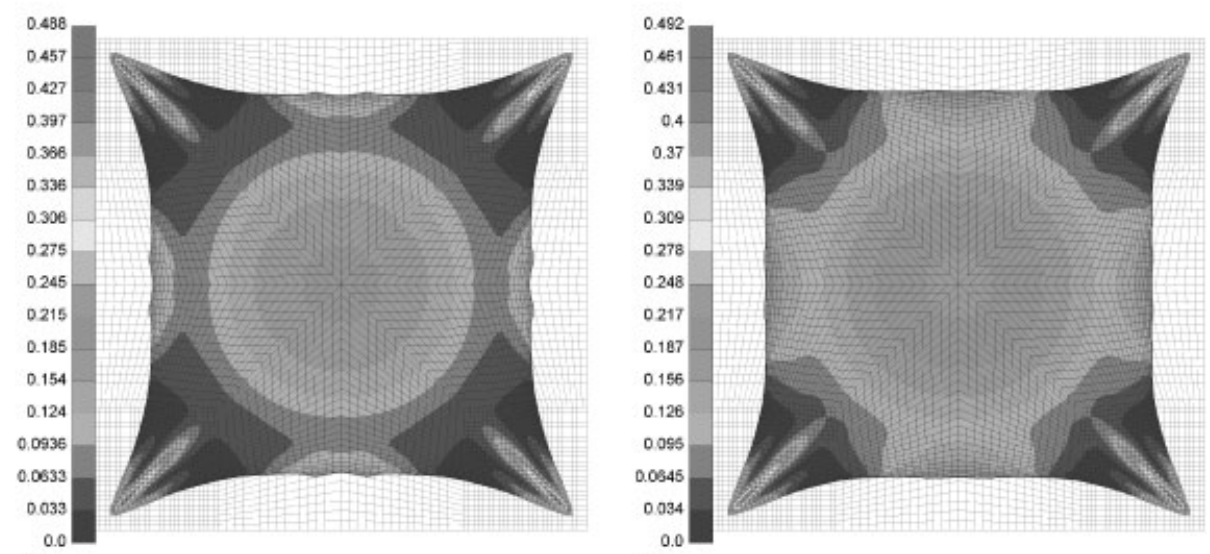

(e)

(f)

Figure 7. Simply-supported plate—deformed configurations (top view) for load factors: (a) $f=285.4$; (b) $f=386.8$; (c) $f=479.9$; (d) $f=557.6$; (e) $f=600.7$; and (f) $f=635.5$. 

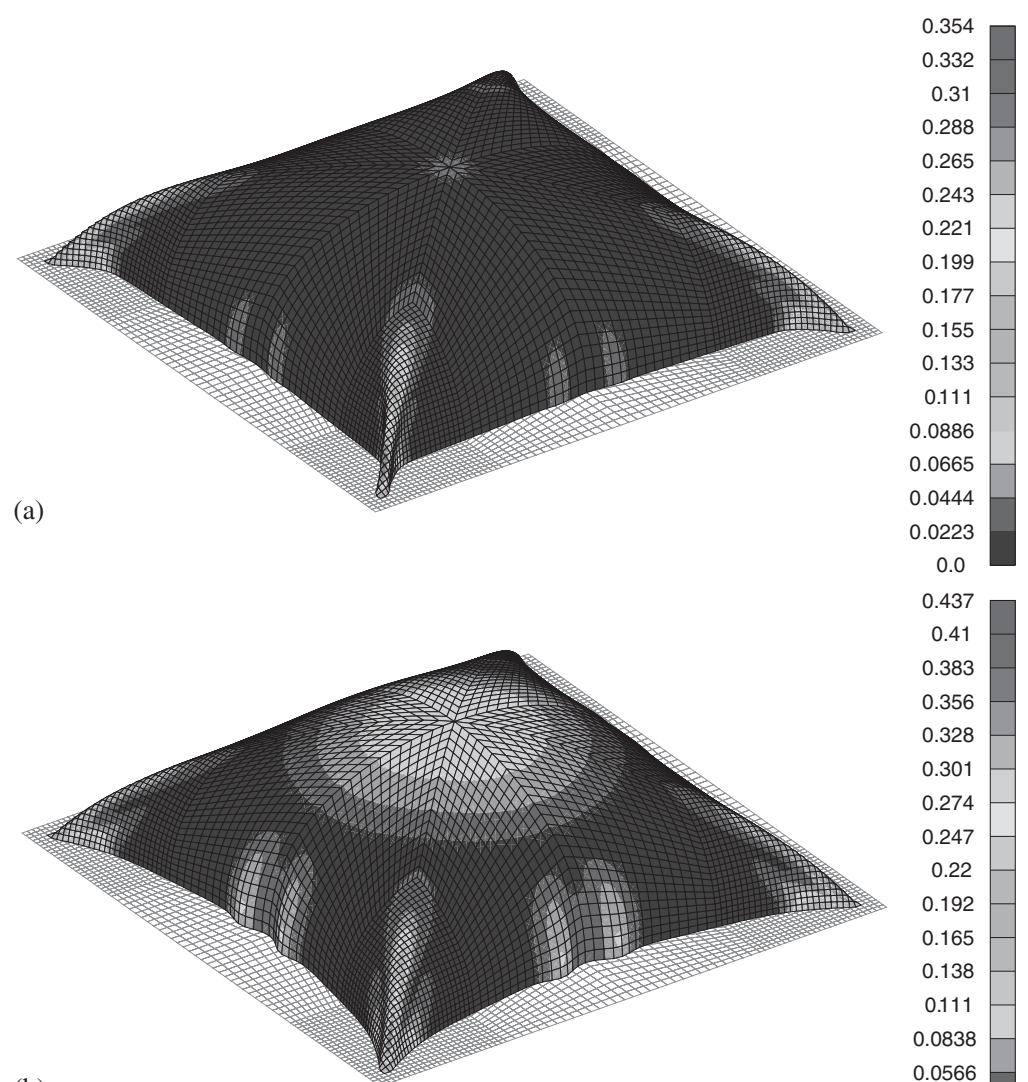

0.437

0.383

0.356

0.328

0.301

0.274

0.247

0.22

0.192

0.165

0.138

0.111

0.0838

0.0566

0.0295

0.0

0.492

0.461

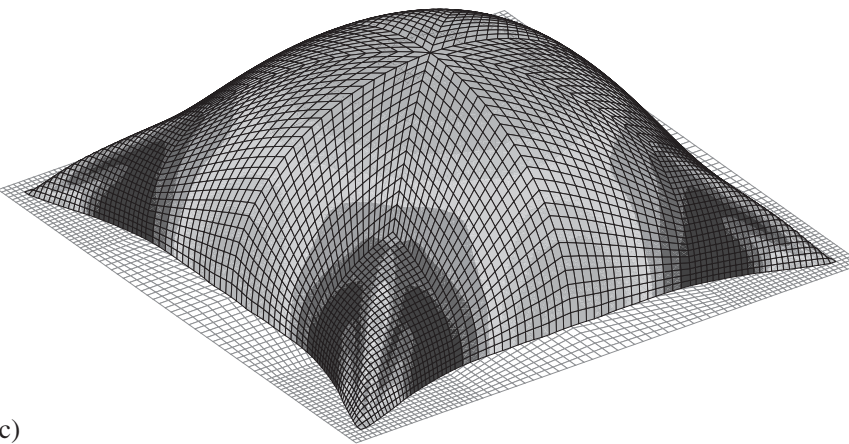

0.431

Figure 8. Simply-supported plate-deformed configurations (dimetric perspective) and equivalent plastic strain for load factors: (a) $f=285.4$; (b) $f=479.9$; and (c) $f=635.5$. 


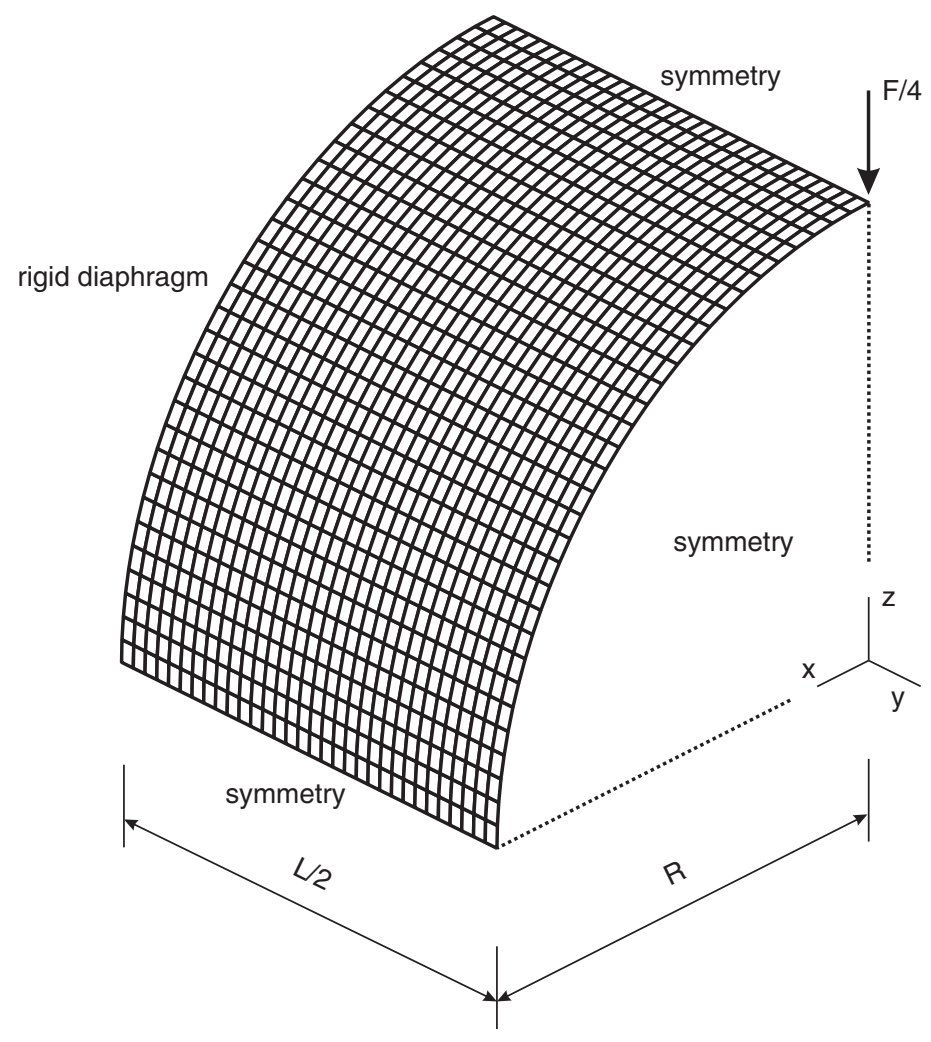

Figure 9. Pinched cylinder-initial configuration with geometry, loading and boundary conditions.

In Figure 10 the displacement of the loaded node is monitored and plotted against the total load level $F$ in Figure 9. For the same mesh, results coming from the presented elastoplastic formulation applied to the $S 4 E 6 P 5$ EAS shell element are compared to the solid-shell formulation proposed by Miehe [61] and to the ' 5 parameter' shell formulation of Eberlein and Wriggers [78].

The results are almost coincident in the major displacement range, with the present element being slightly more flexible for displacements greater than 175 consistent unities (being in accordance with results obtained by Wriggers et al. [88] for a 3D formulation not shown in the figure). Even dealing with a load control procedure, convergence is easily achieved, with the whole displacement path being covered in 108 (automatically incremented) load steps. For the sake of completeness, evolution of the equivalent plastic strain, in successive deformation states, is shown in Figure 11.

\subsection{Anisotropic elasto-plastic constitutive behaviour}

5.2.1. Hydro-bulge forming of a circular plate (bulge test). This example is a well known and useful test to determine stress vs strain curves as well as forming limit diagrams for a given material, being also adopted on the validation of anisotropic models. In this case, and although 


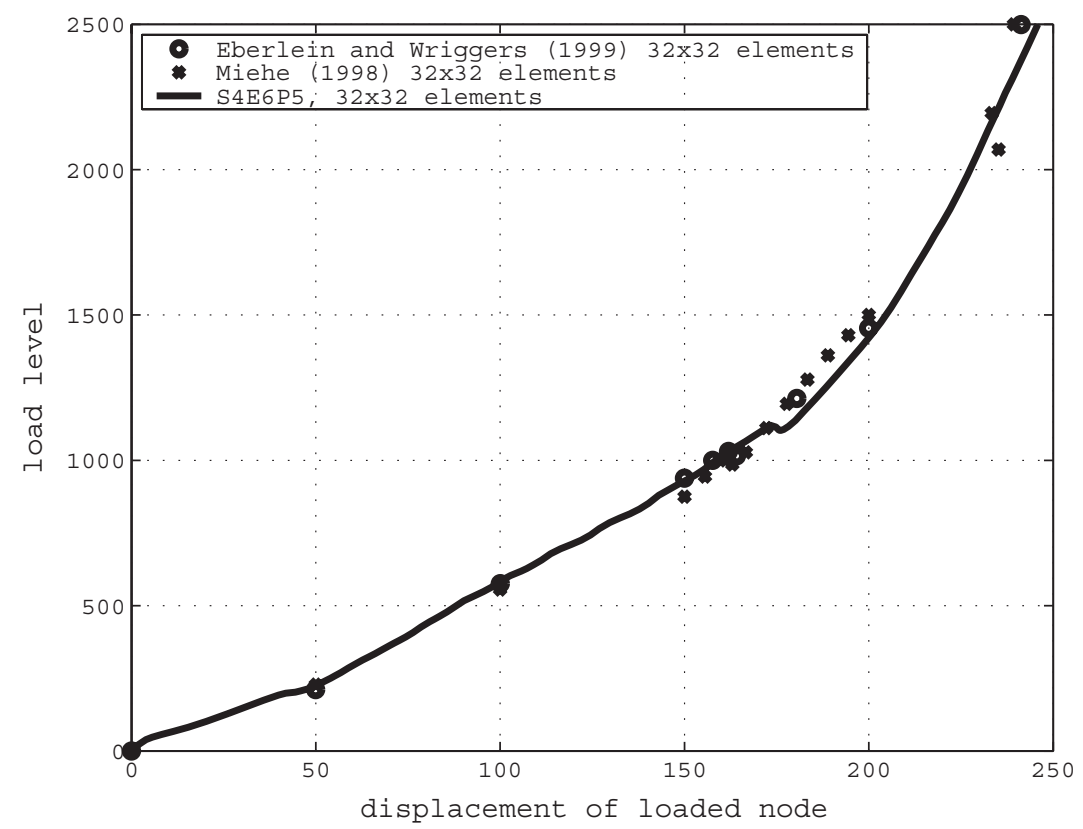

Figure 10. Pinched cylinder-deflection of the loaded node as a function of the external force $(F)$.

the geometric, loading and boundary conditions could induce symmetric strain contours with deformation, anisotropic properties of the 2008-T4 aluminium alloy analysed will inevitably lead to differences from purely isotropic plasticity models. The chosen anisotropic constitutive model follows the criterion proposed by Barlat et al. [74] for aluminium alloys.

The bulge forming starts with a circular plate with an initial radius $R=81 \mathrm{~mm}$ and initial thickness $a=1.24 \mathrm{~mm}$. Isotropic material properties refer to Young modulus $E=69 \mathrm{GPa}$. Poisson coefficient $v=0.33$, isotropic hardening factor $H_{\text {iso }}=0.0$, initial yield stress value of $\sigma_{y 0}=185.0 \mathrm{MPa}$, saturation yield stress value of $\sigma_{y \infty}=408.0 \mathrm{MPa}$ and exponential hardening factor $n=6.14$. All these terms are interrelated in the evolution law for the yield stress level, as a function of the equivalent plastic strain value, in the form

$$
\sigma_{y}=\sigma_{y 0}+H_{\text {iso }} \varepsilon^{p}+\left(\sigma_{y \infty}-\sigma_{y 0}\right)\left(1-\mathrm{e}^{-n \varepsilon^{p}}\right)
$$

Anisotropic parameters, in turn, are given as the values for the constant parameters to be used in the criterion proposed by Barlat et al. [74], being numerically equal to $C_{1}=1.223$, $C_{2}=1.014, C_{3}=0.986, C_{4}=1.0, C_{5}=1.0, C_{6}=1.0$ and $m=11.0$, for the present example $[91,92]$.

The adopted finite element mesh consists of 392 S4E6P5 shell elements, following the mesh distribution in Figure 12, where one-fourth of the plate is represented due to symmetry reasons. For each element, five Gauss points over the thickness direction were adopted. The adopted finite element model is a simplified one, since no contact between the blank-holder and the plate (blank) was taken into account. Instead, the borders of the plate are considered clamped, with the hydrostatic pressure being represented by an uniform pressure load over the plate surface, with nominal value of $p_{0}=4 \mathrm{MPa}$. 
(a)

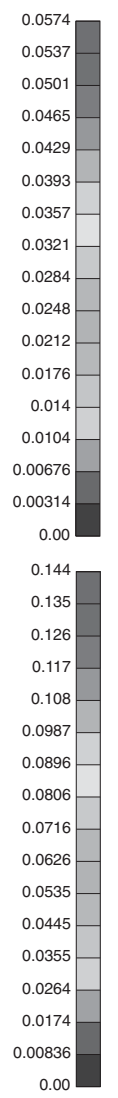

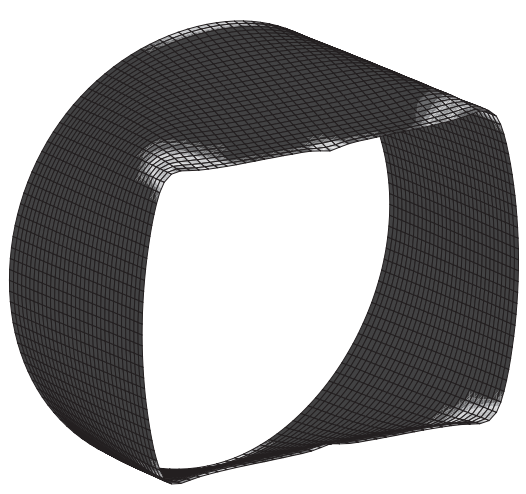

equivalent plastic strain levels

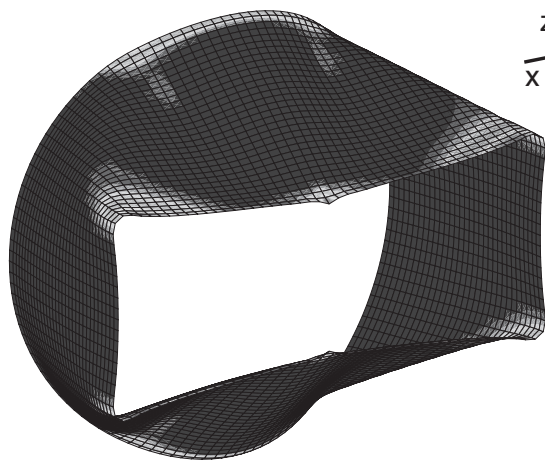

(c)

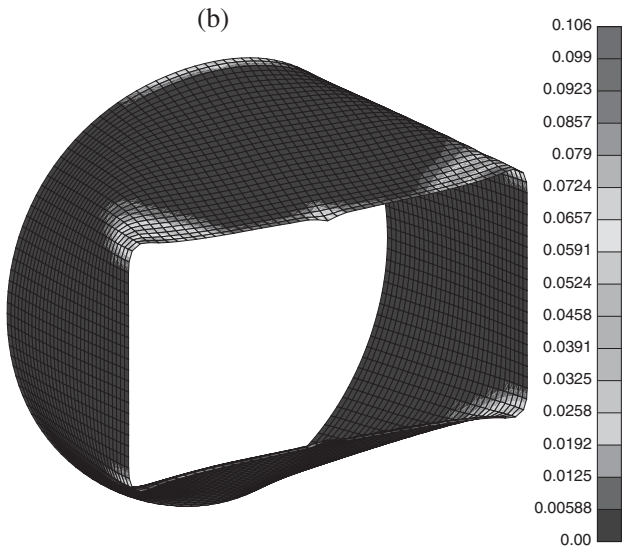

0.357
0.335
0.312
0.29
0.268
0.245
0.223
0.201
0.178
0.156
0.133
0.111
0.0886
0.0662
0.0438
0.0214
0.00

Figure 11. Pinched cylinder-equivalent plastic strain levels for displacement values of: (a) 101.2; (b) 151.3 ; (c) 200.0; and (d) 246.8 consistent unities.

Starting with the imposition of an isotropic von Mises material model, it is possible to establish a set of comparisons between the present shell formulation against results obtained with shell elements from Abaqus finite-element commercial code (shell elements $S 4, S 4 R$ and $S 4 R 5)$. Shell elements in Abaqus code also encompassed five Gauss points along the thickness direction, in accordance to what was done with the present shell element. The analysis of the out-of-plane apex point displacement evolution, with increasing pressure levels, led to the graph represented in Figure 13.

It is interesting to note that the present shell formulation is able to avoid locking effects for the set of boundary, geometry and loading conditions. Additionally, for the present example, the methodology behind the enhanced shell element has not revealed sensitivity to the mesh distortion (namely on the apex zone), which is frequently a strong pitfall for enhanced-based finite elements in the literature. The results obtained with the present finite elements are close to those obtained with Abaqus commercial code. Nevertheless, deviations appear between the simulation results with the $S 4 E 6 P 5$ finite element and the experimental ones. 


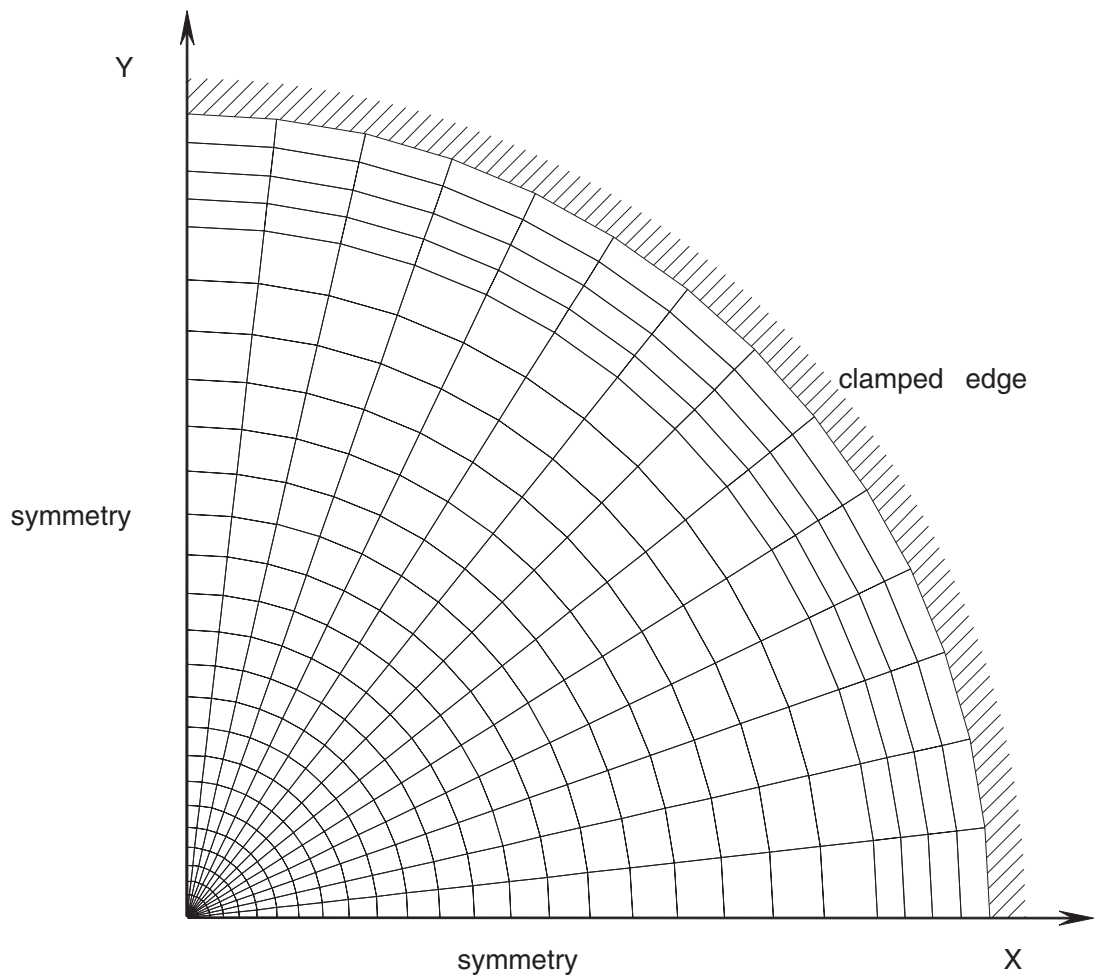

Figure 12. Hydro-bulge forming—-mesh and boundary conditions adopted.

In order to assess the importance of the constitutive law on the quality of the solution, results with the presented enhanced assumed strain shell element along with the anisotropic criterion of Barlat et al. [74] are considered. Experimental results (including anisotropic effects) give a final pole displacement of approximately $23 \mathrm{~mm}$ [91]. For the present EAS shell element, results including the anisotropic criterion are reproduced in Figure 14 and compared to experimental data. At the final pressure level, the EAS shell formulation led to a pole displacement of $23.6 \mathrm{~mm}$, which is in good agreement with the experimental solution, and considerably higher than the analogous result considering an isotropic yield criterion.

For the sake of completeness, equivalent plastic strain contours are presented for specimens with rolling directions at $0^{\circ}$ and $45^{\circ}$ relative to a reference direction $O X$. On algorithm terms, the local axis $\left(\mathbf{r}^{1}\right)$ of each Gauss point is initially taken along these directions, at the start of the numerical process. Subsequent deformation will drive the evolution of the local triad, as described before. For a rolling direction of $0^{\circ}$ with $O X$ direction, the contour plots of the equivalent plastic strains are reproduced in Figure 15, for the maximum pressure level. For a rolling direction of $45^{\circ}$ relative to the reference direction, contour plots of the equivalent plastic strain now change, as can be seen in Figure 16. From this figure, it is visible the lack of concentricity of the contour curves, as well as the influence of measurements along distinct rolling directions, as expected when considering anisotropic constitutive evolution laws. 


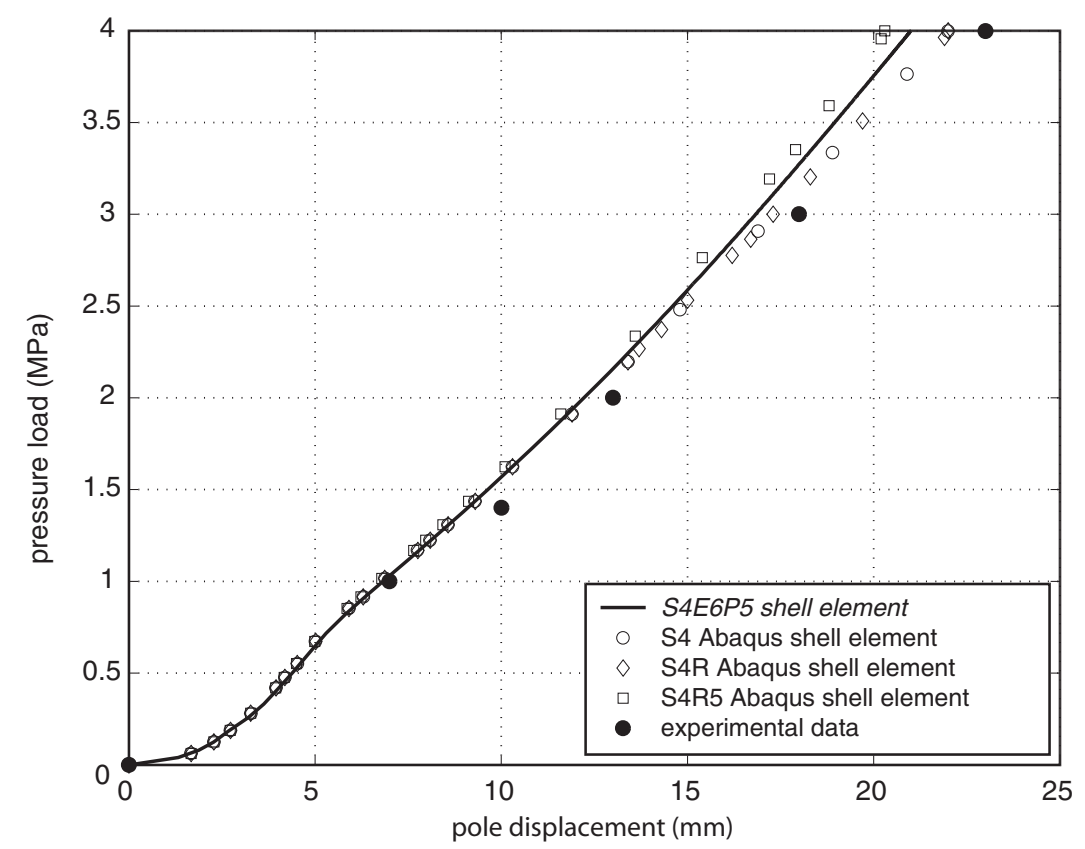

Figure 13. Hydro-bulge forming-results for S4E6P5 EAS shell element for the isotropic constitutive evolution.

5.2.2. Forming of elliptical cross-section pressure vessel heads. In this example, the simulation of the hydro-bulge forming of a pressure vessel head is carried out. The main data for the analysis come from experimental specifications that can be found in Reference [93], dealing with the integral hydro-bulge forming (IHBF) technology, originally proposed for the manufacturing of medium to large radius pressure vessel heads [94, 95].

The motivation in accounting for the present example relies on the fact that, for a given level of forming pressure applied to the initial blank, wrinkles might appear in the deformed part during the manufacturing process. This phenomenon, verified experimentally, is of transient character, tending to disappear with further increase of the fluid pressure applied to the undeformed part. From the simulation standpoint, and as shown here, reproduction of the onset of wrinkles is highly dependent on the constitutive model employed.

Isotropic-based plasticity models cannot reproduce the instability conditions leading to the wrinkles. More interesting, nevertheless, is that planar anisotropic criteria, such as those coming from the works of Hill [73] and Barlat et al. [74], also fails. Wrinkles appearance can be reproduced, on the other hand, with the implementation of a normal anisotropic criterion.

Following Reference [93], a schematic representation of the geometry of the initial (undeformed) part is represented in Figure 17, for a blank thickness of $3 \mathrm{~mm}$. The mesh is distinct to the one adopted in Reference [93], and models one fourth of the whole initial part, due to symmetry reasons. The finite element model is supposed to be clamped on its outer edge, and is supposed to represent a circular central flat plate joined (by welding) to a ring outer zone, built up from the cutting of a pre-bent tube. The whole structure is subjected 


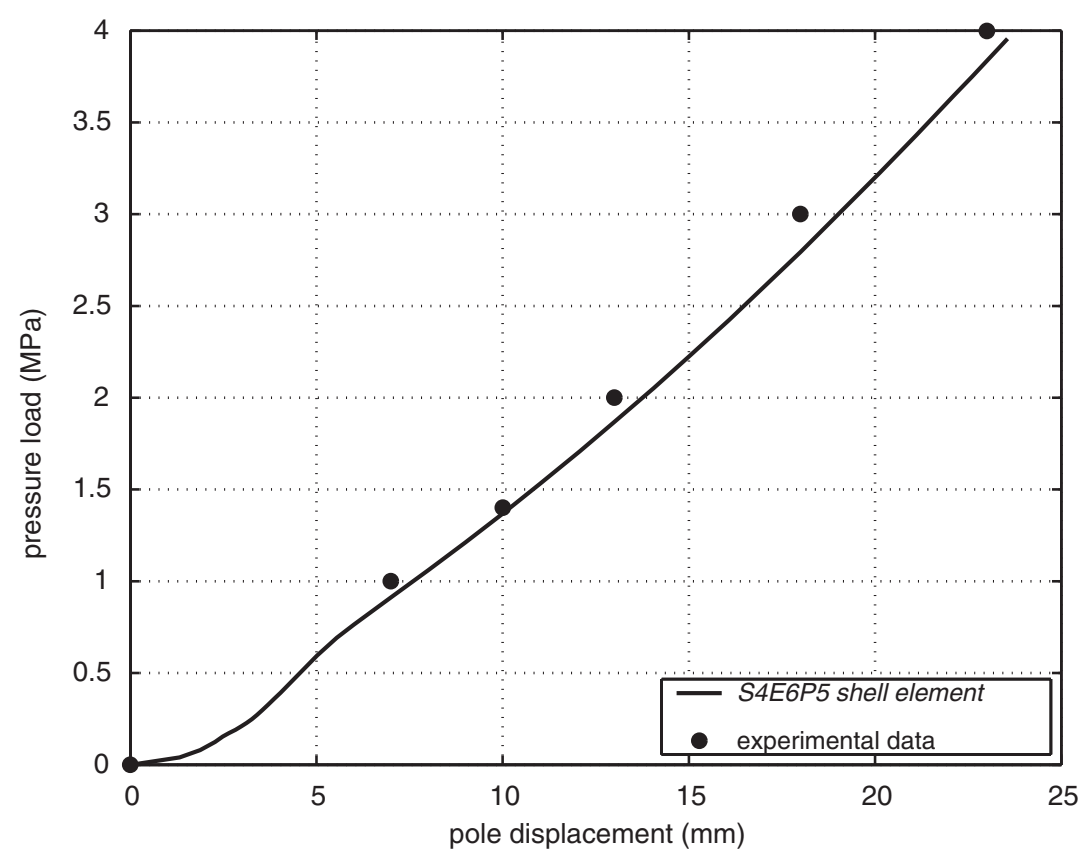

Figure 14. Hydro-bulge forming-results for S4E6 P5 EAS shell element, considering an anisotropic yield criterion.

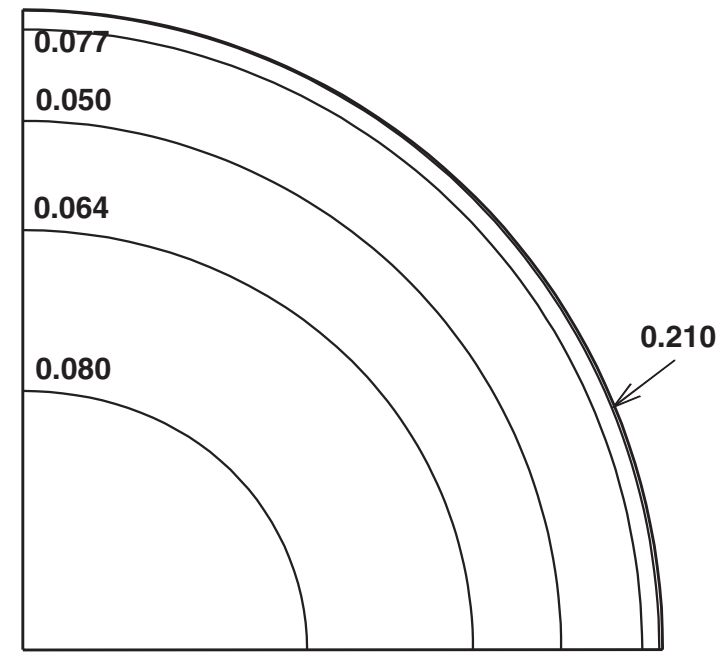

Figure 15. Hydro-bulge forming-equivalent plastic strain distribution for a rolling direction at $0^{\circ}$, considering an anisotropic yield criterion and a pressure level of $4 \mathrm{MPa}$. 


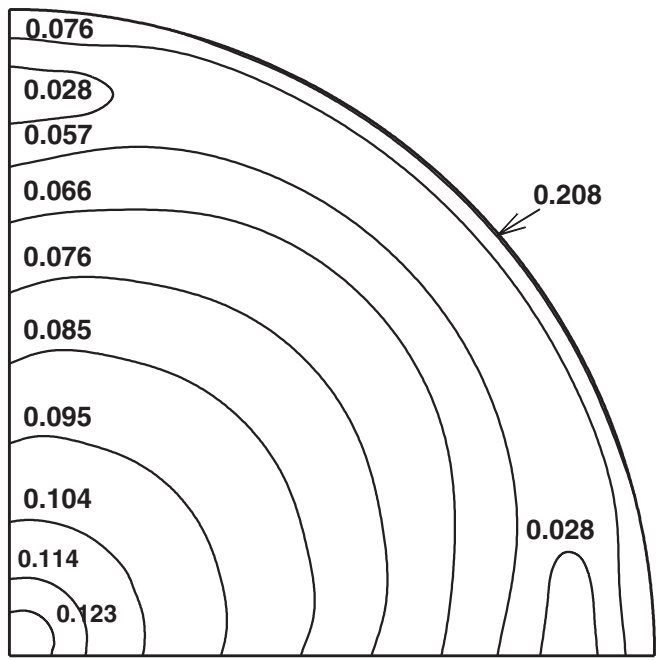

Figure 16. Hydro-bulge forming-equivalent plastic strain distribution for a rolling direction at $45^{\circ}$, considering an anisotropic yield criterion and a pressure level of $4 \mathrm{MPa}$.

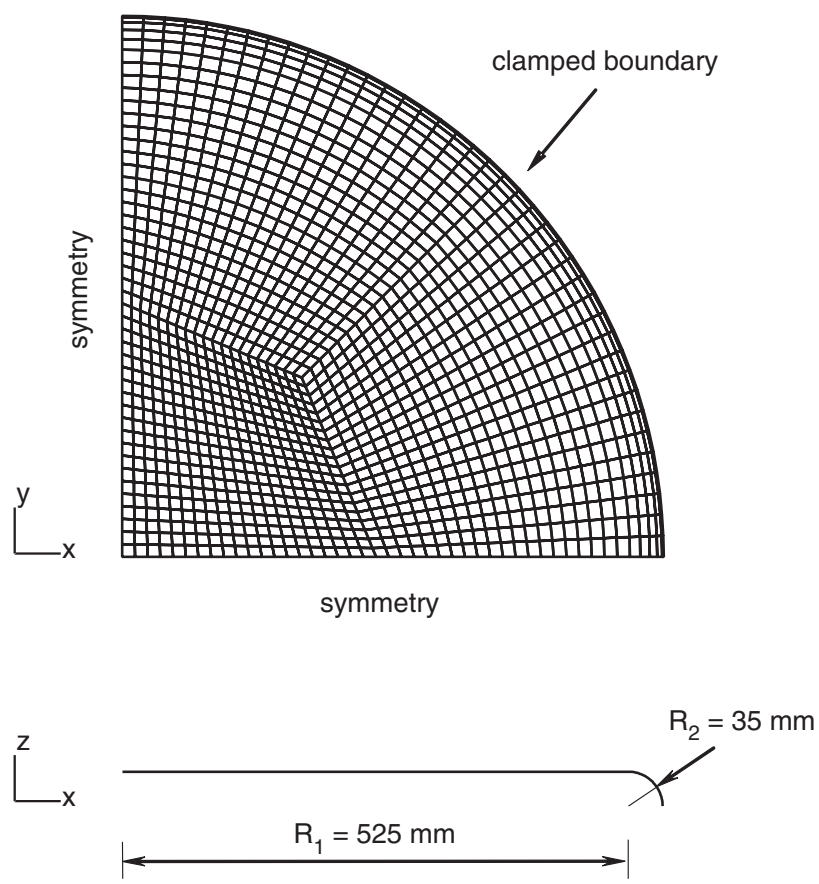

Figure 17. Pressure vessel head-schematic representation of the undeformed (initial) part, along with the mesh and boundary conditions adopted. 

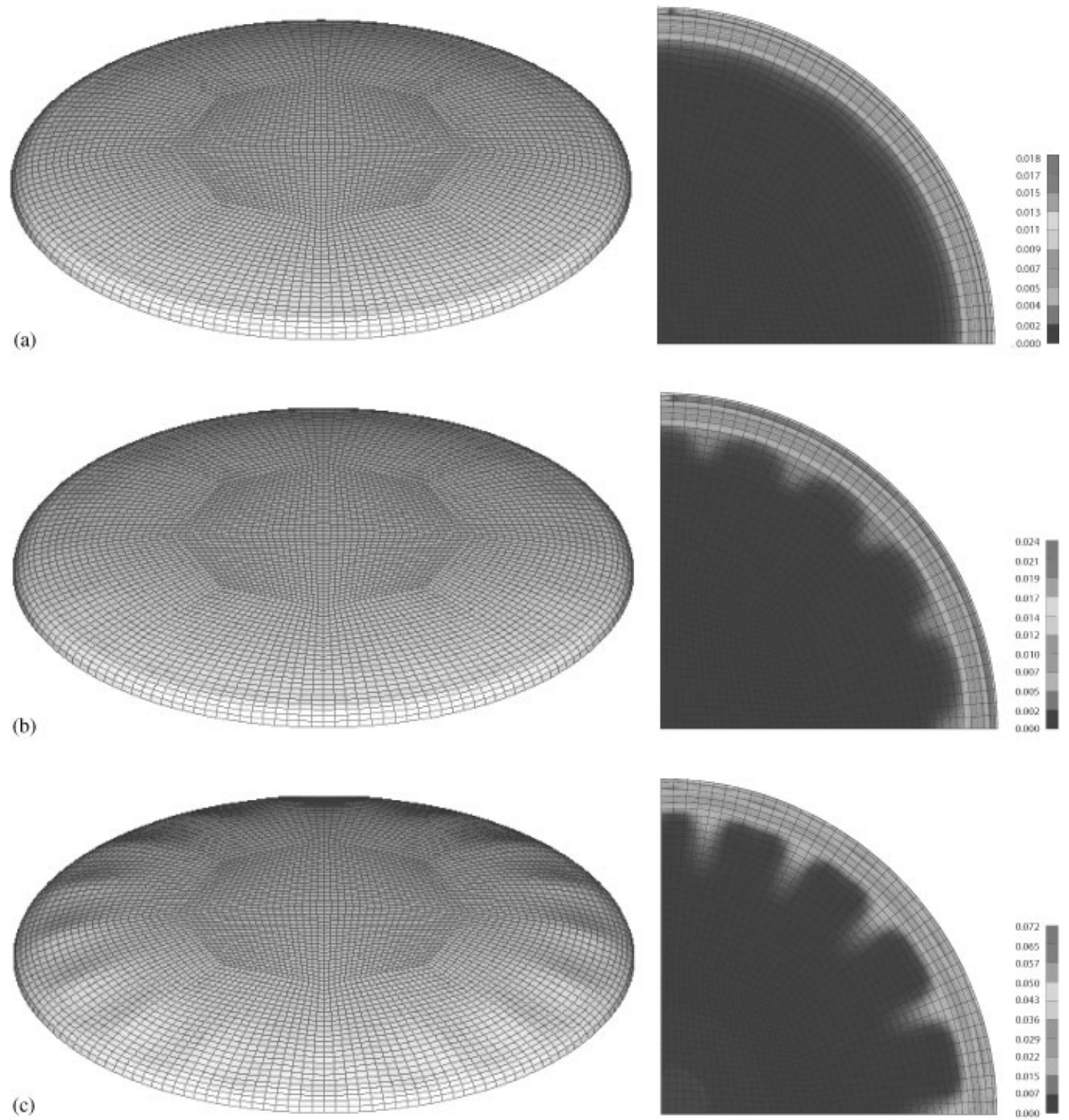

Figure 18. Pressure vessel head-deformed configuration and evolution of equivalent plastic strain for pressure values: (a) $0.18 \mathrm{MPa}$; (b) $0.2 \mathrm{MPa}$; and (c) $0.4 \mathrm{MPa}$.

to pressure on its internal face, with the corresponding deformed form showing an elliptical cross-sectional pattern.

Departing from Reference [93], however, an aluminium alloy similar to the last example is employed here in order to attain a higher number of wrinkles than those obtained in the latter reference (who used steel as the tested material). The material properties for the von Mises and Barlat yield criteria are the same as those used in the last example. Constants for the 

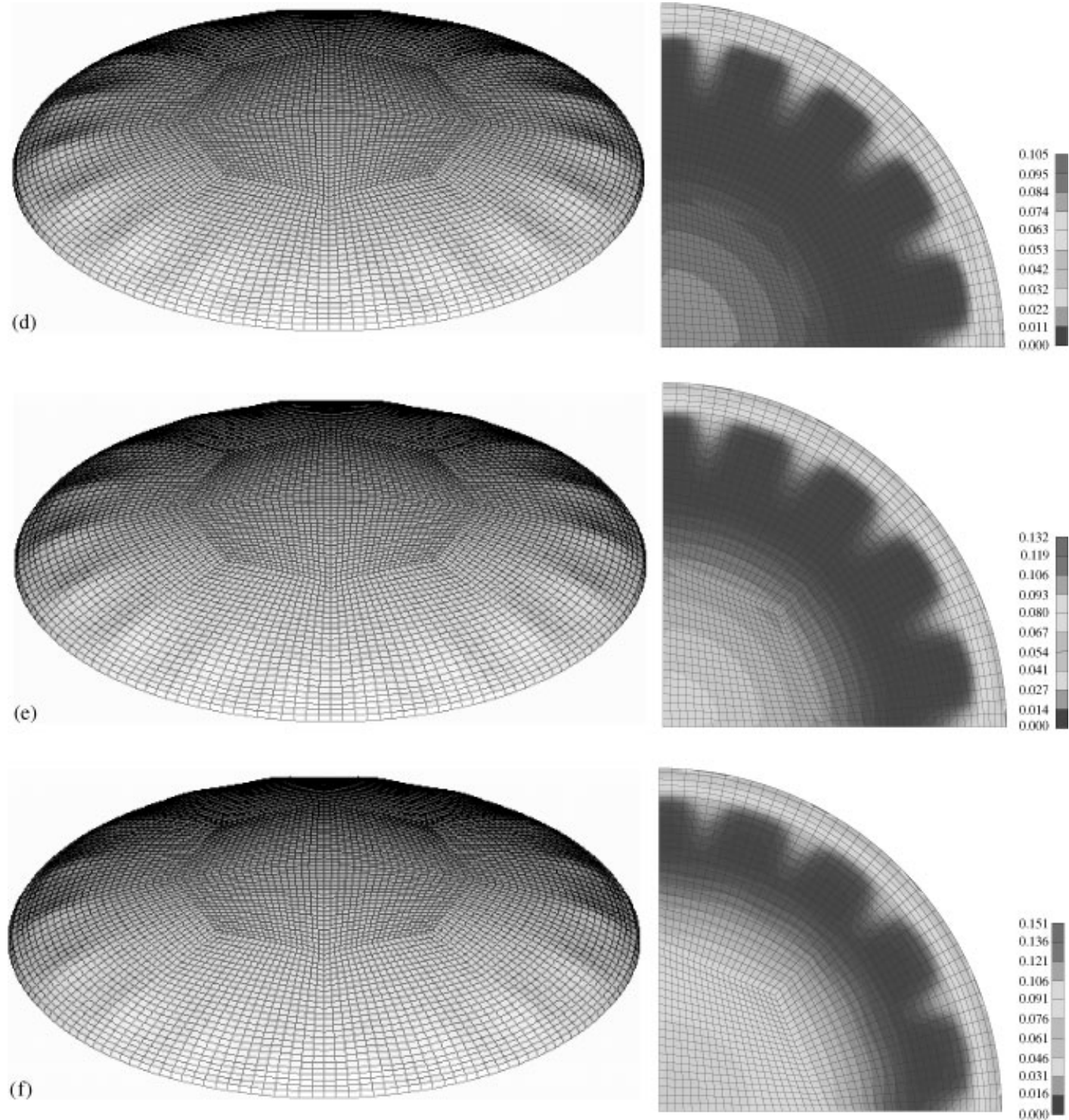

Figure 19. Pressure vessel head (cont.)—deformed configuration and evolution of equivalent plastic strain for pressure values: (d) $0.6 \mathrm{MPa}$; (e) $0.8 \mathrm{MPa}$; and (f) $1.0 \mathrm{MPa}$.

Hill criterion for anisotropic parameters for planar anisotropy are $R_{0}=0.8, R_{45}=0.6$ and $R_{90}=0.7$, while for the normal anisotropy the equivalent (resultant) coefficient is $R=0.675$. The maximum attained pressure load in the simulation (enough to induce the appearance of wrinkles) is equal to $p_{0}=1 \mathrm{MPa}$. All the before-referred constitutive models were implemented and tested, along with their ability to reproduce physical phenomena attained experimentally. 
For the present case (aluminium alloy part), and as said before, only the normal anisotropy yield criterion is able to predict the appearance of the wrinkles along the perimeter of the deformed part. Although the induced equivalent plastic strain level is not high, its gradient is visible over the deformed vessel head, as reproduced in Figures 18 and 19.

For a pressure level of approximately 0.4 MPa, wrinkles initiation is visible, remaining stabilized until an internal pressure value of 1.0 MPa. For higher loads, the wrinkles tend to disappear (with the spread of plasticity on the internal part of the vessel head) and a smooth final elliptical cross-sectional shape is attained. Similar conclusions were also reported in Reference [93] for steel vessel heads.

\section{CONCLUSIONS}

The present work represents the closure of the research line initiated by the authors back in Reference [29], and dealing with the treatment of transverse shear locking in degenerated bilinear shell elements, solely resorting to the application of the EAS method. Finite displacement and rotations algorithmic treatment are carried out here, considering small to moderate strains. The additive dealing of non-additive rotation degrees-of-freedom is described in detail. Furthermore, implementation of isotropic as well as anisotropic yield criteria is dealt with. In the end, an improved EAS-based thin-shell finite element formulation is achieved, involving a minimum set of enhanced variables at each element level, when compared to previous works. The finite element formulation, along with the implemented non-linear geometric and constitutive models, have led to good results when tested in simulation and experimental benchmark problems.

\section{APPENDIX}

Explicit expressions for the convective displacement-based strain tensor components, before the introduction of the enhanced transverse shear assumed strain approach:

$$
\begin{aligned}
E_{\xi^{1} \xi^{1}}^{u}= & \left(\frac{\partial x^{1}}{\partial \xi^{1}} \frac{\partial N}{\partial \xi^{1}}\right) u^{1}+\left(\frac{\partial x^{2}}{\partial \xi^{1}} \frac{\partial N}{\partial \xi^{1}}\right) u^{2}+\left(\frac{\partial x^{3}}{\partial \xi^{1}} \frac{\partial N}{\partial \xi^{1}}\right) u^{3} \\
& +\left(\frac{a}{2} \xi^{3} \frac{\partial N}{\partial \xi^{1}}\left(\frac{\partial x^{1}}{\partial \xi^{1}} v^{11}+\frac{\partial x^{2}}{\partial \xi^{1}} v^{12}+\frac{\partial x^{3}}{\partial \xi^{1}} v^{13}\right)\right) \theta^{1} \\
& -\left(\frac{a}{2} \xi^{3} \frac{\partial N}{\partial \xi^{1}}\left(\frac{\partial x^{1}}{\partial \xi^{1}} v^{21}+\frac{\partial x^{2}}{\partial \xi^{1}} v^{22}+\frac{\partial x^{3}}{\partial \xi^{1}} v^{23}\right)\right) \theta^{2} \\
E_{\xi^{2} \xi^{2}}^{u}= & \left(\frac{\partial x^{1}}{\partial \xi^{2}} \frac{\partial N}{\partial \xi^{2}}\right) u^{1}+\left(\frac{\partial x^{2}}{\partial \xi^{2}} \frac{\partial N}{\partial \xi^{2}}\right) u^{2}+\left(\frac{\partial x^{3}}{\partial \xi^{2}} \frac{\partial N}{\partial \xi^{2}}\right) u^{3} \\
& +\left(\frac{a}{2} \xi^{3} \frac{\partial N}{\partial \xi^{2}}\left(\frac{\partial x^{1}}{\partial \xi^{2}} v^{11}+\frac{\partial x^{2}}{\partial \xi^{2}} v^{12}+\frac{\partial x^{3}}{\partial \xi^{2}} v^{13}\right)\right) \theta^{1}
\end{aligned}
$$




$$
\begin{aligned}
& -\left(\frac{a}{2} \xi^{3} \frac{\partial N}{\partial \xi^{2}}\left(\frac{\partial x^{1}}{\partial \xi^{2}} v^{21}+\frac{\partial x^{2}}{\partial \xi^{2}} v^{22}+\frac{\partial x^{3}}{\partial \xi^{2}} v^{23}\right)\right) \theta^{2} \\
& E_{\xi^{3} \xi^{3}}^{u}=\left(\frac{a}{2} N\left(\frac{\partial x^{1}}{\partial \xi^{3}} v^{11}+\frac{\partial x^{2}}{\partial \xi^{3}} v^{12}+\frac{\partial x^{3}}{\partial \xi^{3}} v^{13}\right)\right) \theta^{1} \\
& -\left(\frac{a}{2} N\left(\frac{\partial x^{1}}{\partial \xi^{3}} v^{21}+\frac{\partial x^{2}}{\partial \xi^{3}} v^{22}+\frac{\partial x^{3}}{\partial \xi^{3}} v^{23}\right)\right) \theta^{2} \\
& 2 E_{\xi^{1} \xi^{2}}^{u}=\left(\frac{\partial x^{1}}{\partial \xi^{1}} \frac{\partial N}{\partial \xi^{2}}+\frac{\partial x^{1}}{\partial \xi^{2}} \frac{\partial N}{\partial \xi^{1}}\right) u^{1}+\left(\frac{\partial x^{2}}{\partial \xi^{1}} \frac{\partial N}{\partial \xi^{2}}+\frac{\partial x^{2}}{\partial \xi^{2}} \frac{\partial N}{\partial \xi^{1}}\right) u^{2} \\
& +\left(\frac{\partial x^{3}}{\partial \xi^{1}} \frac{\partial N}{\partial \xi^{2}}+\frac{\partial x^{3}}{\partial \xi^{2}} \frac{\partial N}{\partial \xi^{1}}\right) u^{3} \\
& +\left(\frac{a}{2} \xi^{3} \frac{\partial N}{\partial \xi^{2}}\left(\frac{\partial x^{1}}{\partial \xi^{1}} v^{11}+\frac{\partial x^{2}}{\partial \xi^{1}} v^{12}+\frac{\partial x^{3}}{\partial \xi^{1}} v^{13}\right)\right. \\
& \left.+\frac{a}{2} \xi^{3} \frac{\partial N}{\partial \xi^{1}}\left(\frac{\partial x^{1}}{\partial \xi^{2}} v^{11}+\frac{\partial x^{2}}{\partial \xi^{2}} v^{12}+\frac{\partial x^{3}}{\partial \xi^{2}} v^{13}\right)\right) \theta^{1} \\
& -\left(\frac{a}{2} \xi^{3} \frac{\partial N}{\partial \xi^{2}}\left(\frac{\partial x^{1}}{\partial \xi^{1}} v^{21}+\frac{\partial x^{2}}{\partial \xi^{1}} v^{22}+\frac{\partial x^{3}}{\partial \xi^{1}} v^{23}\right)\right. \\
& \left.+\frac{a}{2} \xi^{3} \frac{\partial N}{\partial \xi^{1}}\left(\frac{\partial x^{1}}{\partial \xi^{2}} v^{21}+\frac{\partial x^{2}}{\partial \xi^{2}} v^{22}+\frac{\partial x^{3}}{\partial \xi^{2}} v^{23}\right)\right) \theta^{2} \\
& 2 E_{\xi^{1} \xi^{3}}^{u}=\left(\frac{\partial x^{1}}{\partial \xi^{1}} \frac{\partial N}{\partial \xi^{3}}+\frac{\partial x^{1}}{\partial \xi^{3}} \frac{\partial N}{\partial \xi^{1}}\right) u^{1}+\left(\frac{\partial x^{2}}{\partial \xi^{1}} \frac{\partial N}{\partial \xi^{3}}+\frac{\partial x^{2}}{\partial \xi^{3}} \frac{\partial N}{\partial \xi^{1}}\right) u^{2} \\
& +\left(\frac{\partial x^{3}}{\partial \xi^{1}} \frac{\partial N}{\partial \xi^{3}}+\frac{\partial x^{3}}{\partial \xi^{3}} \frac{\partial N}{\partial \xi^{1}}\right) u^{3} \\
& +\left(\frac { a } { 2 } \left(v^{11}\left(N \frac{\partial x^{1}}{\partial \xi^{1}}+\xi^{3} \frac{\partial N}{\partial \xi^{1}} \frac{\partial x^{1}}{\partial \xi^{3}}\right)+v^{12}\left(N \frac{\partial x^{2}}{\partial \xi^{1}}+\xi^{3} \frac{\partial N}{\partial \xi^{1}} \frac{\partial x^{2}}{\partial \xi^{3}}\right)\right.\right. \\
& \left.\left.+v^{13}\left(N \frac{\partial x^{3}}{\partial \xi^{1}}+\xi^{3} \frac{\partial N}{\partial \xi^{1}} \frac{\partial x^{3}}{\partial \xi^{3}}\right)\right)\right) \theta^{1} \\
& -\left(\frac { a } { 2 } \left(v^{21}\left(N \frac{\partial x^{1}}{\partial \xi^{1}}+\xi^{3} \frac{\partial N}{\partial \xi^{1}} \frac{\partial x^{1}}{\partial \xi^{3}}\right)+v^{22}\left(N \frac{\partial x^{2}}{\partial \xi^{1}}+\xi^{3} \frac{\partial N}{\partial \xi^{1}} \frac{\partial x^{2}}{\partial \xi^{3}}\right)\right.\right. \\
& \left.\left.+v^{23}\left(N \frac{\partial x^{3}}{\partial \xi^{1}}+\xi^{3} \frac{\partial N}{\partial \xi^{1}} \frac{\partial x^{3}}{\partial \xi^{3}}\right)\right)\right) \theta^{2}
\end{aligned}
$$




$$
\begin{aligned}
2 E_{\xi^{2} \xi^{3}}^{u}= & \left(\frac{\partial x^{1}}{\partial \xi^{2}} \frac{\partial N}{\partial \xi^{3}}+\frac{\partial x^{1}}{\partial \xi^{3}} \frac{\partial N}{\partial \xi^{2}}\right) u^{1}+\left(\frac{\partial x^{2}}{\partial \xi^{2}} \frac{\partial N}{\partial \xi^{3}}+\frac{\partial x^{2}}{\partial \xi^{3}} \frac{\partial N}{\partial \xi^{2}}\right) u^{2} \\
& +\left(\frac{\partial x^{3}}{\partial \xi^{2}} \frac{\partial N}{\partial \xi^{3}}+\frac{\partial x^{3}}{\partial \xi^{3}} \frac{\partial N}{\partial \xi^{2}}\right) u^{3} \\
& +\left(\frac { a } { 2 } \left(v^{11}\left(N \frac{\partial x^{1}}{\partial \xi^{2}}+\xi^{3} \frac{\partial N}{\partial \xi^{2}} \frac{\partial x^{1}}{\partial \xi^{3}}\right)+v^{12}\left(N \frac{\partial x^{2}}{\partial \xi^{2}}+\xi^{3} \frac{\partial N}{\partial \xi^{2}} \frac{\partial x^{2}}{\partial \xi^{3}}\right)\right.\right. \\
& \left.\left.+v^{13}\left(N \frac{\partial x^{3}}{\partial \xi^{2}}+\xi^{3} \frac{\partial N}{\partial \xi^{2}} \frac{\partial x^{3}}{\partial \xi^{3}}\right)\right)\right) \theta^{1} \\
& -\left(\frac { a } { 2 } \left(v^{21}\left(N \frac{\partial x^{1}}{\partial \xi^{2}}+\xi^{3} \frac{\partial N}{\partial \xi^{2}} \frac{\partial x^{1}}{\partial \xi^{3}}\right)+v^{22}\left(N \frac{\partial x^{2}}{\partial \xi^{2}}+\xi^{3} \frac{\partial N}{\partial \xi^{2}} \frac{\partial x^{2}}{\partial \xi^{3}}\right)\right.\right. \\
& \left.\left.+v^{23}\left(N \frac{\partial x^{3}}{\partial \xi^{2}}+\xi^{3} \frac{\partial N}{\partial \xi^{2}} \frac{\partial x^{3}}{\partial \xi^{3}}\right)\right)\right) \theta^{2}
\end{aligned}
$$

\section{ACKNOWLEDGEMENTS}

Funding by Ministério da Ciência e do Ensino Superior, FCT and FSE, Portugal, under grant PRAXIS $\mathrm{XXI} / \mathrm{BD} / 21662 / 99$; as well as the funding by FEDER, under grant POCTI/EME/47289/2002 and POSI SFRH/BD/13013/2003 are gratefully acknowledged.

\section{REFERENCES}

1. Ahmad S, Irons BM, Zienkiewicz OC. Analysis of thick and thin shell structures by curved finite elements. International Journal for Numerical Methods in Engineering 1970; 2:419-451.

2. Krakeland B. Large displacement analysis of shells considering elastic-plastic and elasto-viscoplastic materials. Report No. 776, Norwegian Institute of Technology, University of Trondheim, Norway, 1977.

3. Ramm E. A plate/shell Element for large deflections and rotations. In Formulations and Computational Algorithms in Finite Element Analysis, Bathe KJ, Oden JT, Wunderlich W (eds). MIT Press: Cambridge, MA, 1977.

4. Zienkiewicz OC, Taylor RL, Too JM. Reduced integration techniques in general analysis of plates and shells. International Journal for Numerical Methods in Engineering 1979; 3:275-290.

5. Bathe KJ, Bolourchi S. A geometric and material nonlinear plate and shell element. Computers and Structures 1980; 11:23-48.

6. Hughes TJR, Liu WK. Nonlinear finite element analysis of shells: part I. Three-dimensional shells. Computer Methods in Applied Mechanics and Engineering 1981; 26:331-362.

7. Hughes TJR, Tezduyar TE. Finite elements based upon Mindlin plate theory with particular reference to the four-node bilinear isoparametric element. Journal of Applied Mechanics (ASME) 1981; 48:587-596.

8. Liu WK, Lam D, Law SE, Belytschko T. Resultant stress degenerated shell element. Computer Methods in Applied Mechanics and Engineering 1986; 55:259-300.

9. Yang HTY, Saigal S, Masud A, Kapania RK. A survey of recent shell finite elements. International Journal for Numerical Methods in Engineering 2000; 47:101-127.

10. Chapelle D, Bathe KJ. Fundamental considerations for the finite element analysis of shell structures. Computers and Structures 1998; 66:19-36. 
11. Bathe KJ, Iosilevich A, Chapelle D. An evaluation of the MITC shell elements. Computers and Structures 2000; 75:1-30.

12. Chapelle D, Bathe KJ. The mathematical shell model underlying general shell elements. International Journal for Numerical Methods in Engineering 2000; 48:289-313.

13. Chapelle D. Some new results and current challenges in the finite element analysis of shells. Acta Numerica 2001; 10:215-250.

14. Bathe KJ, Chapelle D, Lee PS. A shell problem 'highly sensitive' to thickness changes. International Journal for Numerical Methods in Engineering 2003; 57:1039-1052.

15. Chapelle D, Bathe KJ. The Finite Element Analysis of Shells Fundamentals. Springer: Berlin, Heidelberg, New York, 2003.

16. Hughes TJR, Cohen M, Haroun M. Reduced and selective integration techniques in finite element analysis of plates. Nuclear Engineering Design 1978; 46:203-222.

17. Dvorkin E, Bathe KJ. A continuum mechanics based four-node shell element for general nonlinear analysis. Engineering Computations 1984; 1:77-88.

18. Bathe KJ, Dvorkin E. A formulation of general shell elements-the use of mixed interpolation of tensorial components. International Journal for Numerical Methods in Engineering 1986; 22:697-722.

19. MacNeal RH. A simple quadrilateral shell element. Computers and Structures 1978; 8:175-183.

20. MacNeal RH. Derivation of element stiffness matrices by assumed strain distribution. Nuclear Engineering Design 1982; 703:3-12.

21. Park KC. Improved strain interpolation for curved $C^{0}$ elements. International Journal for Numerical Methods in Engineering 1986; 22:281-288.

22. Park KC, Stanley GM. A curved $C^{0}$ shell element based on assumed natural coordinate strains. Journal of Applied Mechanics 1986; 53:278-290.

23. Stanley GM, Park KC, Hughes TJR. Continuum-based resultant shell elements. In Finite Element Methods for Plate and Shell Structures, Hughes TJR, Hinton E (eds). Pineridge Press: Swansea, 1986; 1-45.

24. Simo JC, Rifai MS. A class of mixed assumed strain methods and the method of incompatible modes. International Journal for Numerical Methods in Engineering 1990; 29:1595-1638.

25. Simo JC, Armero F. Geometrically non-linear enhanced strain mixed methods and the method of incompatible modes. International Journal for Numerical Methods in Engineering 1992; 33:1443-1449.

26. Simo JC, Armero F, Taylor RL. Improved versions of assumed enhanced strain tri-linear elements for 3D finite deformation problems. Computer Methods in Applied Mechanics and Engineering 1993; 110:359-386.

27. Andelfinger U, Ramm E. EAS-Elements for two-dimensional, three-dimensional, plate and shells and their equivalence to HR-elements. International Journal for Numerical Methods in Engineering 1993; 36:1413-1439.

28. Büchter N, Ramm E, Roehl D. Three-dimensional extension of nonlinear shell formulation based on the enhanced assumed strain concept. International Journal for Numerical Methods in Engineering 1994; 37: $2551-2568$.

29. César de Sá JMA, Natal Jorge RM, Fontes Valente RA, Areias PMA. Development of shear locking-free shell elements using an enhanced assumed strain formulation. International Journal for Numerical Methods in Engineering 2002; 53:1721-1750.

30. César de Sá JMA, Natal Jorge RM. New enhanced strain elements for incompressible problems. International Journal for Numerical Methods in Engineering 1999; 44:229-248.

31. César de Sá JMA, Areias PMA, Natal Jorge RM. Quadrilateral elements for the solution of elasto-plastic finite strain problems. International Journal for Numerical Methods in Engineering 2001; 51:883-917.

32. Fontes Valente RA, Natal Jorge RM, Cardoso RPR, César de Sá JMA, Grácio JJ. On the use of an enhanced transverse shear strain shell element for problems involving large rotations. Computational Mechanics 2003; 30:286-296.

33. Fontes Valente RA. Developments on shell and solid-shell finite elements technology in nonlinear continuum mechanics. Ph.D. Dissertation, University of Porto, Portugal, 2004.

34. Alves de Sousa RJ, Natal Jorge RM, Fontes Valente RA, César de Sá JMA. A new volumetric and shear locking-free 3D enhanced strain element. Engineering Computations 2003; 20:896-925.

35. Fontes Valente RA, Alves de Sousa RJ, Natal Jorge RM. An enhanced strain 3D element for large deformation elastoplastic thin-shell applications. Computational Mechanics 2004; 34:38-52.

36. Areias PMA, César de Sá JMA, Conceição António CA, Fernandes AA. Analysis of 3D problems using a new enhanced strain hexahedral element. International Journal for Numerical Methods in Engineering 2003; 58: $1637-1682$. 
37. Bathe KJ. Finite Element Procedures (2nd edn). Prentice-Hall: New Jersey, 1996.

38. ABAQUS Theory Manual. Version 6.3. Hibbit, Karlsson \& Sorensen, Inc.: Rhode-Island, USA, 2002.

39. Hughes TJR. The Finite Element Method: Linear Static and Dynamic Finite Element Analysis (2nd edn). Dover Editions: Toronto, 2000.

40. Yoon JW, Yang DY, Chung K. Elasto-plastic finite element method based on incremental deformation theory and continuum based shell elements for planar anisotropic sheet materials. Computer Methods in Applied Mechanics and Engineering 1999; 174:23-56.

41. Cardoso RPR, Yon JW, Grácio JJA, Barlat F, César de Sá JMA. Development of a one point quadrature shell element for nonlinear applications with contact and anisotropy. Computer Methods in Applied Mechanics and Engineering 2002; 191:5177-5206.

42. Saleeb AF, Chang TY, Graf W, Yingyeunyong S. A hybrid/mixed model for non-linear shell analysis and its applications to large-rotation problems. International Journal for Numerical Methods in Engineering 1990; 29:407-446.

43. Basar Y, Weicher D. Nonlinear Continuum Mechanics of Solids. Springer: Berlin, Heidelberg, New York, 2000.

44. Bütcher N, Ramm E. Shell theory versus degeneration-a comparison in large rotation finite element analysis. International Journal for Numerical Methods in Engineering 1992; 34:39-59.

45. Betsch P, Manzel A, Stein E. On parameterization of finite rotations in computational mechanics. A classification of concepts with application to smooth shells. Computer Methods in Applied Mechanics and Engineering 1998; 155:273-305.

46. Doyle JF. Nonlinear Analysis of Thin-Walled Structures Statics: Dynamics and Stability. Springer: Berlin, Heidelberg, New York, 2001.

47. Crisfield MA. Non-Linear Finite Element Analysis of Solids and Structures, vol. 2. Advanced Topics. Wiley: West Sussex, 1997.

48. Franca LP. An algorithm to compute the square root of a $3 \times 3$ positive definite matrix. Computers and Mathematics with Applications 1989; 18:459-466.

49. Qin Z, Chen Z. Large deformation analysis of shells with finite element method based on the SR decomposition theorem. Computers and Structures 1988; 30:957-961.

50. Peng X, Crisfield MA. A consistent corotational formulation for shells using the constant stress/constant moment triangle. International Journal for Numerical Methods in Engineering 1992; 35:1829-1847.

51. Jiang L, Chernuka MW. A simple four-noded corotational shell element for arbitrarily large rotations. Computers and Structures 1994; 53:1123-1132.

52. Jiang L, Chernuka MW. A co-rotational, updated lagrangean formulation for geometrically nonlinear finite element analysis of shell structures. Finite Elements in Analysis and Design 1994; 18:129-140.

53. Moita GF, Crisfield MA. A finite element formulation for 3D continua using the corotational technique. International Journal for Numerical Methods in Engineering 1996; 39:3775-3792.

54. Belytschko T, Liu WK, Moran B. Nonlinear Finite Elements for Continua and Structures. Wiley: West Sussex, England, 2000.

55. Masud A, Tham CL, Liu WK. A stabilized 3D co-rotational formulation for geometrically nonlinear analysis of multi-layered composite shells. Computational Mechanics 2000; 26:1-12.

56. Masud A, Tham CL. Three-dimensional corotational framework for finite deformation elasto-plastic analysis of multilayered composite shells. AIAA American Institute of Aeronautics and Astronautics Journal 2000; 38:1-8.

57. Bischoff M, Ramm E. Shear deformable shell elements for large strains and rotations. International Journal for Numerical Methods in Engineering 1997; 40:4427-4449.

58. Klinkel S, Wagner W. A geometrical nonlinear brick element based on the EAS-method. International Journal for Numerical Methods in Engineering 1997; 40:4529-4545.

59. Klinkel S, Gruttman F, Wagner W. A continuum based 3D-shell element for laminated structures. Computers and Structures 1999; 74:43-62.

60. Vu-Quoc L, Tan XG. Optimal solid shells for non-linear analysis of multilayer composites. Part I: statics. Computer Methods in Applied Mechanics and Engineering 2003; 192:975-1016.

61. Miehe C. A theoretical and computational model for isotropic elastoplastic stress analysis in shells at large strains. Computer Methods in Applied Mechanics and Engineering 1998; 155:193-233.

62. Freischlager C, Schweizerhof K. On a systematic development of trilinear three-dimensional solid elements based on Simo's enhanced strain formulation. International Journal of Solids and Structures 1996; 33: 2993-3017. 
63. Roehl D, Ramm E. Large elasto-plastic finite element analysis of solids and shells with the enhanced assumed strain concept. International Journal of Solids and Structures 1996; 33:3215-3237.

64. Flanagan DP, Taylor LM. An accurate numerical algorithm for stress integration with finite rotations. Computer Methods in Applied Mechanics and Engineering 1987; 62:305-320.

65. Marsden JE, Hughes TJR. Mathematical Foundations of Elasticity. Dover Publications: Mineola, USA, 1994.

66. Doghri I. Mechanics of Deformable Solids-Linear, Nonlinear, Analytical and Computational Aspects. Springer: Berlin, Heidelberg, New York, 2000.

67. Hughes TJR. Numerical implementation of constitutive models: rate independent deviatoric plasticity. In Theoretical Foundations for Large-Computations for Non-Linear Material Behavior, Nemat-Nasser $\mathrm{S}$ et al. (eds). Martinus Nijhoff Publishers: Dordrecht, The Netherlands, 1984.

68. Key SW, Krieg RD. On the numerical implementation of inelastic time dependent and time independent finite strain constitutive equations in structural mechanics. Computer Methods in Applied Mechanics and Engineering 1982; 33:439-452.

69. Pinsky PM, Ortiz M, Pister KS. Numerical integration of rate constitutive equations in finite deformation analysis. Computer Methods in Applied Mechanics and Engineering 1983; 40:137-158.

70. Ortiz M, Popov EP. Accuracy and stability of integration algorithms for elastoplastic constitutive relations. International Journal for Numerical Methods in Engineering 1985; 21:1561-1576.

71. Ramm E, Matzenmiller A. Consistent linearization in elasto-plastic shell analysis. Engineering Computations 1988; 5:289-299.

72. Brank B, Peric D, Damjanic FB. On large deformations of thin elasto-plastic shells: implementation of a finite rotation model for a quadrilateral shell element. International Journal for Numerical Methods in Engineering 1997; 40:689-726.

73. Hill R. A theory of the yielding and plastic flow of anisotropic metals. Proceedings of the Royal Society of London Series A: Mathematical and Physical Sciences 1948; 193:281-297.

74. Barlat F, Lege DJ, Brem JC. A six-component yield function for anisotropic metals. International Journal of Plasticity 1991; 7:693-712.

75. Chroscielewski J, Makowski J, Stumpf H. Genuinely resultant shell finite elements accounting for geometric and material non-linearities. International Journal for Numerical Methods in Engineering 1992; 35:63-94.

76. Ibrahimbegović A, Frey F. Stress resultant geometrically nonlinear shell theory with drilling rotation Part II: computational aspects. Computer Methods in Applied Mechanics and Engineering 1994; 188:285-308.

77. Betsch P, Gruttmann F, Stein E. A 4-node finite shell element for the implementation of general hyperelastic 3D-elasticity at finite strains. Computer Methods in Applied Mechanics and Engineering 1996; 130:57-79.

78. Eberlein R, Wriggers P. Finite element concepts for finite elastoplastic strains and isotropic stress response in shells: theoretical and computational analysis. Computer Methods in Applied Mechanics and Engineering 1999; 171:243-279.

79. Li M, Zhan F. The finite deformation theory for beam, plate and shells. Part V: the shell element with drilling degrees of freedom based on biot strain. Computer Methods in Applied Mechanics and Engineering 2000; 189:743-759.

80. Crisfield MA. A fast incremental/iterative solution procedure that handle 'snap-through'. Computers and Structures 1981; 13:55-62.

81. Crisfield MA. An arc-length method including line searches and accelerations. International Journal for Numerical Methods in Engineering 1983; 19:1269-1289.

82. de Souza Neto EA, Feng YT. On the determination of the path direction for arc-length methods in the presence of bifurcations and 'snap-backs'. Computer Methods in Applied Mechanics and Engineering 1999; 179:81-89.

83. Betsch P, Stein E. Numerical implementation of multiplicative elasto-plasticity into assumed strain elements with application to shells at large strains. Computer Methods in Applied Mechanics and Engineering 1999; 179:215-245.

84. Hauptmann R, Schweizerhof K, Doll S. Extension of the solid-shell concept for application to large elastic and large elastoplastic deformations. International Journal for Numerical Methods in Engineering 2000; 49: $1121-1141$.

85. Doll S, Schweizerhof K, Hauptmann R, Freischläger C. On volumetric locking of low-order solid and solid-shell elements for finite elastoviscoplastic deformations and selective reduced integration. Engineering Computations 2000; 17:874-902.

86. Harnau M, Schweizerhof K. About linear and quadratic 'solid-shell' elements at large deformations. Computers and Structures 2002; 80:805-817. 
87. Simo JC, Kennedy JG. On a stress resultant geometrically exact shell model. Part V: nonlinear plasticityformulation and integration algorithms. Computer Methods in Applied Mechanics and Engineering 1992; 96: $133-171$.

88. Wriggers P, Eberlein R, Reese S. A comparison of three-dimensional continuum and shell elements for finite plasticity. International Journal of Solids and Structures 1996; 33:3309-3326.

89. Sorić J, Montag U, Krätzig WB. An efficient formulation of integration algorithms for elastoplastic shell analysis based on layered finite element approach. Computer Methods in Applied Mechanics and Engineering 1997; 148:315-328.

90. Sansour C, Kollmann FG. Families of 4-node and 9-node finite elements for a finite deformation shell theory: An assessment of hybrid stress, hybrid strain and enhanced strain elements. Computational Mechanics 2000; 24:435-447.

91. Yoon JW. Finite element formulation based on incremental deformation theory for sheet metal forming of planar anisotropic materials. Ph.D. Dissertation, Korea Advanced Institute of Science and Technology (KAIST), South Korea, 1996.

92. Cardoso RPR. Development of one point quadrature shell elements with anisotropic material models for sheet metal forming analysis. Ph.D. Dissertation, University of Aveiro, Portugal, 2002.

93. Zhang SH, Danckert J, Nielsen KB. Integral hydro-bulge forming of pressure vessel heads. Journal of Materials Processing Technology 1999; 86:184-189.

94. Wang ZR, Wang T, Kang DC, Zhang SH, Fang Y. The technology of the hydro-bulging of whole spherical vessels and experimental analysis. Journal of Mechanical Working Technology 1989; 18:85-94.

95. Zhang SH, Wang ZR. Research into the dieless hydroforming of double-layer spherical vessels. International Journal of Pressure Vessels and Piping 1994; 60:145-149. 